\title{
Instability of plumes driven by localized heating
}

\author{
Juan M. Lopez ${ }^{1, \dagger}$ and Francisco Marques ${ }^{2}$ \\ ${ }^{1}$ School of Mathematical and Statistical Sciences, Arizona State University, Tempe, AZ 85287, USA \\ ${ }^{2}$ Departament de Física Aplicada, Universidad Politècnica de Catalunya, Barcelona 08034, Spain
}

(Received 19 April 2013; revised 9 July 2013; accepted 7 October 2013;

first published online 13 November 2013)

Plumes due to localized buoyancy sources are of wide interest owing to their prevalence in many situations. This study investigates the transition from laminar to turbulent dynamics. Several experiments have reported that this transition is sensitive to external perturbations. As such, a well-controlled set-up has been chosen for our numerical study, consisting of a localized heat source at the bottom of an enclosed cylinder whose walls are all maintained at a fixed uniform temperature, except for the localized heat source. At moderate Rayleigh numbers $R a$, the flow consists of a steady, axisymmetric purely poloidal plume. On increasing $R a$, the flow undergoes a supercritical Hopf bifurcation to an axisymmetric 'puffing' plume, where a vortex ring is periodically emitted from the localized heater. At higher $R a$, this state becomes unstable to a sequence of symmetry-breaking bifurcations, going through a quasiperiodic 'fluttering' stage where the axisymmetric rings are tilted, and other states in which the sequence of tilted rings interact with each other. The sequence of symmetry-breaking bifurcations in the transition to turbulence culminates in a torus breakup event in which all the spatial and spatio-temporal symmetries of the system are broken.

Key words: bifurcation, nonlinear instability, plumes/thermals

\section{Introduction}

Plumes due to localized buoyancy sources are of wide interest due to their prevalence in many situations, including fires, chimneys, volcanoes, deep-sea hydrothermal vents and a wide variety of other atmospheric and oceanic situations (Turner 1969; Fay 1973; List 1982; Linden 2000; Woods 2010). Also, plumes play an important role even when the buoyancy source is not localized, such as in Rayleigh-Bénard convection (Siggia 1994; Ahlers 2009; Ahlers, Grossman \& Lohse 2009). The term plume is used to describe the situation where a maintained buoyancy source leads to localized motion of buoyant fluid away from the source. If the source of buoyancy is intermittent, the resulting fluid motions are called puffs. If there is also a source of momentum, the resulting motions are called buoyant jets or buoyant puffs, depending on whether the sources are persistent or intermittent. We are concerned here with plumes.

Early studies of plumes concerned their mixing and dispersion properties. The governing equations were found to be intractable and progress was made by assuming 
far-field similarity in a turbulent state which allowed one to consider averaged quantities. There has been much theoretical interest in thermal plumes since the pioneering theoretical treatment of Morton, Taylor \& Turner (1956). They developed a theory for the entrainment of ambient fluid into a 'turbulent' plume based on the assumption of a self-similar structure of the averaged plume flow, i.e. that the profiles of vertical velocity and buoyancy are similar at all heights. Since then there have been many experimental, theoretical and numerical studies on thermal plumes, but most of them focused on fully turbulent regimes, and on the mixing properties of the plumes.

In this study, we are interested in the transition from laminar to turbulent plumes. In experiments, this transition is found to be sensitive to external perturbations (Gebhart 1973; Pham, Plourde \& Kim 2005; Elicer-Cortés et al. 2006). Therefore a wellcontrolled set-up is crucial to investigate intrinsic instabilities leading to transition, rather than extraneously driven transition. In order to do that, a simple setting has been chosen: a localized heat source at the bottom of an enclosed cylindrical container, the boundaries of which are at a uniform temperature except for the localized heat source. It seems that many experimental as well as numerical studies have been designed to match as closely as possible the conditions under the assumptions of the model of Morton et al. (1956), and this has led to attempts to minimize effects of enclosures. The experiments in large apparatuses have far-field conditions that are difficult to characterize and in some cases difficult to control, while numerically, artificial convective boundary conditions are often employed (Pham, Plourde \& Doan 2007; Plourde et al. 2008). In contrast, the experiments and numerical simulations of Torrance, Orloff \& Rockett (1969) and Torrance \& Rockett (1969) are completely enclosed and very similar to what we consider here. There are also many experimental studies of plumes in enclosures using salt water as a buoyancy source (e.g. Baines \& Turner 1969), but the boundary conditions are qualitatively different from those when buoyancy is due to a local heat source (zero flux rather than constant temperature), and can lead to effects such as the 'filling box' phenomenon.

There are a number of numerical studies of turbulent plumes (e.g. Pham et al. 2005, 2007; Plourde et al. 2008), where numerical noise is imposed on the vertical velocity at a level of $5 \%$ very close to the buoyancy source in order to drive the flow into a turbulent state, as it was found that without noise the solutions remain laminar. As such, there is a lack of understanding of the transitional processes between steady axisymmetric laminar plumes and turbulent plumes. There have also been numerous simulations of axisymmetric plumes (Torrance \& Rockett 1969; Ulucaki 1996; Jiang \& Luo 2000) and planar two-dimensional plumes from a line source of buoyancy (Desrayaud \& Lauriat 1993; Soteriou, Dong \& Cetegen 2002), but even though some of these are in regimes where the solutions are time-dependent, their relevance to the question of transition to three-dimensional turbulent plumes is unclear due to their restrictions to two-dimensional flows.

There has been much interest in understanding the instabilities leading to various three-dimensional time-dependent plume states (e.g. Cetegen, Dong \& Soteriou 1998; Maxworthy 1999; Soteriou et al. 2002; DesJardin, O’Hern \& Tieszen 2004; Williams et al. 2007) with inconsistencies between the various experimental and numerical studies. Of course, the boundary conditions, geometry and symmetries employed in the various studies differ, and while the primary plume formation is not very sensitive to these details, the instabilities are.

In the present study, we have found a sequence of bifurcations/transitions from the conduction-dominated laminar state at low Rayleigh numbers to a chaotic plume at high Rayleigh numbers. The different transitions are associated with breaking the 
(a)

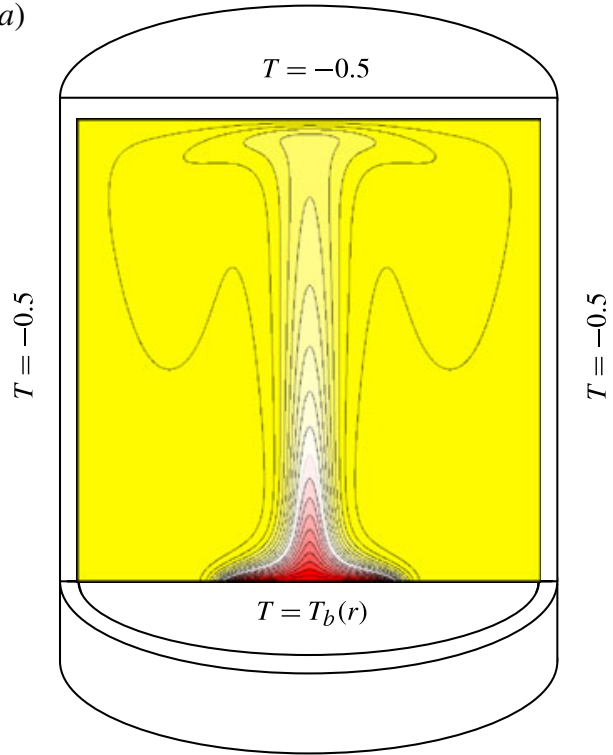

(b)

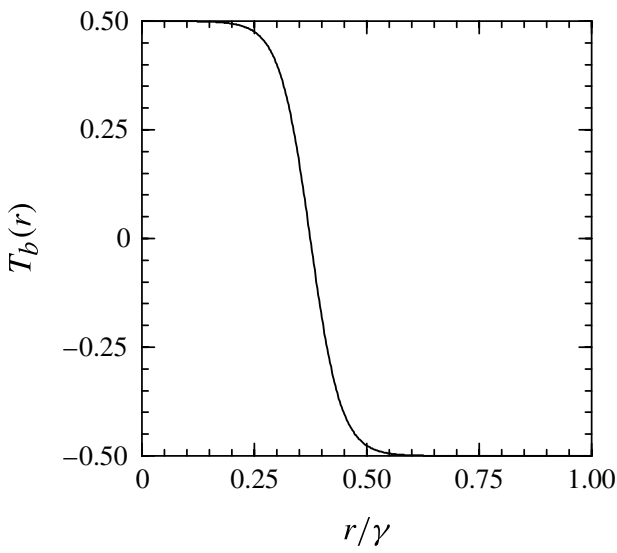

FIGURE 1. (Colour online) (a) Schematic of the apparatus with contours of non-dimensional temperature $T$ showing a laminar thermal plume emerging from the localized heating at the base centre. (b) Radial temperature profile at the bottom wall.

symmetries of the system, and some of these occur over a small range in Rayleigh number. The different bifurcated states that appear upon increasing the Rayleigh number, and their symmetries that strongly influence the nature of the bifurcations, are analysed in detail, using direct numerical simulations of the Navier-Stokes-Boussinesq equations and dynamical systems theory.

\section{Governing equations and numerical technique}

Consider the flow in a circular cylinder, with no-slip boundary conditions, of radius $r_{0}$ and depth $d$. The top endwall and the sidewall are maintained at constant temperature $T_{0}-\Delta T / 2$ while the bottom endwall has a fixed temperature that decreases from $T_{0}+\Delta T / 2$ at the axis to $T_{0}-\Delta T / 2$ at the sidewall; $T_{0}$ is the reference temperature and $\Delta T$ is the temperature difference between the bottom and top endwalls at the axis. Figure 1(a) shows a schematic of the cylinder. The Boussinesq approximation is implemented, treating all fluid properties as constant except for a linear variation of density with temperature in the gravitational buoyancy term. The system is non-dimensionalized using $d$ as the length scale, $d^{2} / v$ as the time scale ( $v$ is the kinematic viscosity), and $v^{2} \rho_{0} / d^{2}$ as the pressure scale $\left(\rho_{0}\right.$ is the density at temperature $T_{0}$ ). The non-dimensional governing equations are:

$$
\begin{aligned}
\left(\partial_{t}+\boldsymbol{u} \cdot \nabla\right) \boldsymbol{u} & =-\nabla p+\nabla^{2} \boldsymbol{u}+\sigma^{-1} \operatorname{RaT} \boldsymbol{e}_{z}, \\
\left(\partial_{t}+\boldsymbol{u} \cdot \nabla\right) T & =\sigma^{-1} \nabla^{2} T, \quad \nabla \cdot \boldsymbol{u}=0,
\end{aligned}
$$

where $\boldsymbol{u}=(u, v, w)$ is the velocity in cylindrical coordinates $(r, \theta, z)$, the corresponding vorticity is $(\xi, \eta, \zeta), p$ is the dynamic pressure, $\boldsymbol{e}_{z}=(0,0,1)$ is the vertical unit vector in the $z$-direction and $T=\left(T_{\text {phys }}-T_{0}\right) / \Delta T$ is the non-dimensional temperature, while $T_{p h y s}$ is the dimensional temperature. 
The non-dimensional parameters are:

$$
\begin{aligned}
\text { Rayleigh number: } & R a=\alpha g d^{3} \Delta T / \kappa \nu, \\
\text { Prandtl number: } & \sigma=v / \kappa, \\
\text { aspect ratio: } & \gamma=r_{0} / d,
\end{aligned}
$$

where $\alpha$ is the coefficient of volume expansion, $\kappa$ is the thermal diffusivity and $g$ is the acceleration due to gravity. The boundary conditions are:

$$
\begin{array}{cll}
r=\gamma: & T=-0.5, & u=v=w=0, \\
z=1: & T=-0.5, & u=v=w=0, \\
z=0: & T=T_{b}(r), & u=v=w=0 .
\end{array}
$$

The temperature profile at the bottom wall is of the form

$$
T_{b}(r)=0.5-\left(1+\exp \left(C\left(r_{d}+0.5 r_{w}-r\right) / r_{w}\right)^{-1}, \quad r \in[0, \gamma] .\right.
$$

By taking $C$ large enough, the temperature is $T \approx 0.5$ for $r \in\left[0, r_{d}\right]$ and $T \approx-0.5$ for $r \in\left[r_{d}+r_{w}, \gamma\right]$. In fact,

$$
T\left(r_{d}\right)=0.5-\epsilon \quad \text { and } \quad T\left(r_{d}+r_{w}\right)=-0.5+\epsilon \quad \text { for } C=2 \ln (-1+1 / \epsilon) .
$$

We have taken $C \approx 7.4$ so that $95 \%$ of the temperature variation takes place over the interval $r \in\left[r_{d}, r_{d}+r_{w}\right]$. Figure $1(b)$ shows the radial temperature profile imposed at the bottom wall, for $C=7.4$ and $r_{d}=r_{w}=\gamma / 4=0.125$ for $\gamma=0.5$, which are typical values for most of the simulations presented here.

The governing equations have been solved using the second-order time-splitting method proposed in Hughes \& Randriamampianina (1998) combined with a pseudospectral method for the spatial discretization, utilizing a Galerkin-Fourier expansion in the azimuthal coordinate $\theta$ and Chebyshev collocation in $r$ and $z$. In order to handle the coordinate singularity at the axis due to the use of polar coordinates, the prescription in Fornberg (1998) has been followed. The expansion for the temperature is given by the real part of

$$
T(r, \theta, z)=\sum_{m=0}^{2 n_{r}+1} \sum_{n=0}^{n_{z}} \sum_{k=-n_{\theta} / 2}^{n_{\theta} / 2-1} T_{m n k} \Xi_{m}(r / \gamma) \Xi_{n}(2 z) \mathrm{e}^{\mathrm{i} k \theta},
$$

where $\Xi_{n}$ is the $n$th Chebyshev polynomial. The expansions for the velocity components $u, v$ and $w$ are analogous. Fornberg's prescription is that $T_{m n k}=w_{m n k}=0$ when $k+m$ is odd, and $u_{m n k}=v_{m n k}=0$ when $k+m$ is even; there are precisely $n_{r}+1$ non-zero coefficients for fixed $n$ and $k$ in (2.7). Due to the expansion (2.7) the origin is never a collocation point, and the equations are solved in the physical domain $(r, \theta, z)=(0, \gamma] \times(0,2 \pi] \times[-1 / 2,1 / 2]$. Following Orszag \& Patera (1983), we have used the combinations $u_{+}=u+\mathrm{i} v$ and $u_{-}=u-\mathrm{i} v$ in order to decouple the linear diffusion terms in the momentum equations. For each Fourier mode, the resulting Helmholtz equations for $T, w, u_{+}$and $u_{-}$have been solved using a diagonalization technique in the two coordinates $r$ and $z$. Fornberg's prescription guarantees the regularity conditions at the origin needed to solve the Helmholtz equations (Mercader, Net \& Falqués 1991).

The spectral solver is based on that described by Mercader, Batiste \& Alonso (2010) and it has recently been tested and used in a wide variety of enclosed cylinder flows with and without temperature gradients (Marques et al. 2007; Lopez \& Marques 


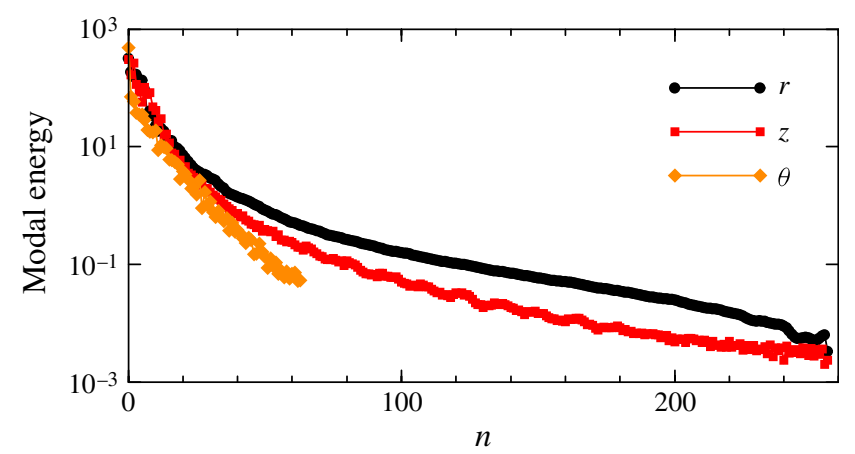

FIGURE 2. (Colour online) Modal kinetic energies in the three coordinate directions as functions of the spectral modes for a solution at $R a=10^{8}$ with $\gamma=0.5, \sigma=7$, and $r_{d}=r_{w}=0.125$.

2009, 2010; Do, Lopez \& Marques 2010; Lopez \& Marques 2011). For the solutions presented here, with $\gamma=0.5$, we have used up to $n_{r}=n_{z}=256$ Chebyshev modes in the radial and axial directions and up to $n_{\theta}=64$ azimuthal Fourier modes. The number of Chebyshev spectral modes is high in order to well-resolve the plume structure, the rapid variation of the temperature at the bottom wall, and the boundary layers forming at the endwalls at the high Rayleigh numbers considered. The spectral resolution for a typical high- $R a$ case is illustrated in figure 2, where the modal kinetic energies in the three coordinate directions as functions of the spectral modes are plotted for a case that has undergone transition at $R a=10^{8}$ with $\gamma=0.5, \sigma=7$, and $r_{d}=r_{w}=0.125$. The solution is well-resolved with at least four orders of magnitude of decay in the modal energies.

It is of interest to consider the vorticity equation, where $\boldsymbol{\nabla} \times \boldsymbol{u}=\boldsymbol{\omega}=(\xi, \eta, \zeta)$ is the vorticity vector,

$$
\left(\partial_{t}+\boldsymbol{u} \cdot \nabla\right) \boldsymbol{\omega}=(\boldsymbol{\omega} \cdot \nabla) \boldsymbol{u}+\nabla^{2} \boldsymbol{\omega}+\sigma^{-1} R a \nabla T \times \boldsymbol{e}_{z} .
$$

The last term on the right-hand side represents the baroclinic production of vorticity. In cylindrical polar coordinates, it is $\sigma^{-1} R a((1 / r) \partial T / \partial \theta,-\partial T / \partial r, 0)$.

\subsection{Symmetries}

The symmetry group of the governing equations and boundary conditions is $O(2)$, corresponding to invariance under arbitrary rotations about the cylinder axis and reflections about any vertical plane through the axis. The symmetries of a physical system have profound implications for the nature of the bifurcations the system can undergo (Chossat \& Lauterbach 2000; Golubitsky \& Stewart 2002; Haragus \& Iooss 2011). The codimension-one bifurcations of fixed points in a generic system are the saddle-node and Hopf bifurcations. These are the bifurcation one can expect if the $O$ (2) symmetry is not broken at the bifurcation. It is also possible to have bifurcations breaking the reflection symmetry but preserving the rotational symmetry $S O(2)$. These bifurcations typically generate swirl (i.e. they have a non-zero azimuthal velocity), and because they break the $Z_{2}$ symmetry reflection they are pitchfork bifurcations. The two bifurcating states are reflection-symmetry related. This is a well-known way to generate swirl via symmetry breaking, already observed in convection problems (Murphy \& Lopez 1984; Massaguer, Mercader \& Net 1990), and recently in localized heating in a cylindrical annulus (Navarro \& Herrero 2011). In the present problem 
there is no swirl generation in the first bifurcation. What we have found is a Hopf bifurcation to a periodic axisymmetric pulsing state preserving $O(2)$ (see $\S 4$ ).

Subsequent transitions correspond to bifurcations of limit cycles with $O(2)$ symmetry. Via analysis of a Poincaré section of the periodic solution, they reduce to the bifurcations of fixed points of maps with $O(2)$ symmetry. The codimensionone bifurcations of fixed points of differential equations with $O(2)$ symmetry are well-understood: for steady bifurcations they are pitchforks-of-revolution, and the time-dependent bifurcations are Hopf bifurcations that result in either rotating waves in which the reflection symmetry is broken, or standing waves that preserve the reflection symmetry (Dangelmayr \& Knobloch 1987; Crawford \& Knobloch 1991; Knobloch 1996). The codimension-one bifurcations of fixed points of maps with $O$ (2) symmetry are also well-known (e.g. Crawford \& Knobloch 1988; Krupa 1990; Marques, Lopez \& Blackburn 2004). The resulting bifurcations bear a strong similarity to the bifurcations of fixed points of differential equations with $O(2)$ symmetry. If axisymmetry is broken and the bifurcating eigenvalue is +1 , then the bifurcation is a pitchfork-of-revolution of limit cycles, the 'revolution' corresponding to the azimuthal phase of the non-axisymmetric pattern. If the bifurcating eigenvalue is -1 , the bifurcation is again a pitchfork-of-revolution of limit cycles, but now the period of the bifurcating limit cycles is doubled. Both bifurcations with eigenvalues \pm 1 preserve the reflection symmetry. If the bifurcation is quasi-periodic (i.e. a second frequency appears following the bifurcation that is generically incommensurate with the limit cycle frequency), then we either have modulated rotating waves (that have broken the reflection symmetry) or quasi-periodic patterns preserving reflection symmetry.

More complicated bifurcations of limit cycles with $O(2)$ symmetry are possible when we are close to codimension-two bifurcations. These may occur due to degeneracies in the normal form (Knobloch 1986; Nagata 1986) or due to mode interactions between closely bifurcating solutions (Armbruster, Guckenheimer \& Holmes 1988). In these cases additional branches of solutions appear at the bifurcation point, consisting of mixed modes that may have all the symmetries of the problem broken, secondary bifurcations to three-tori, and complex dynamics. Since these bifurcations result in the creation of invariant two- or three-tori, the individual solutions on the torus may have all the symmetries broken while the invariant set (the torus) may be invariant under some of the symmetries of the problem. A detailed analysis of this type of symmetry breaking in the individual solution whilst preserving the symmetry of the invariant set in a Taylor-Couette system was presented in Altmeyer et al. (2012). This type of behaviour is observed in the present plume flow (see $\S 5$ ).

The $L_{2}$-norms of the azimuthal Fourier modes (modal kinetic energies) of a given solution,

$$
E_{m}=\frac{1}{2} \int_{z=0}^{z=1} \int_{r=0}^{r=\gamma} \boldsymbol{u}_{m} \cdot \boldsymbol{u}_{m}^{*} r \mathrm{~d} r \mathrm{~d} z,
$$

where $\boldsymbol{u}_{m}$ is the $m$ th Fourier mode of the velocity field and $\boldsymbol{u}_{m}^{*}$ is its complex conjugate, provide a convenient way to characterize the non-axisymmetric states.

\section{Basic state}

The governing equations (2.1)-(2.2) have been integrated forward in time, starting from rest, until an asymptotically steady state has been reached. The localized heating in the centre of the bottom endwall means that there is a non-zero radial temperature 

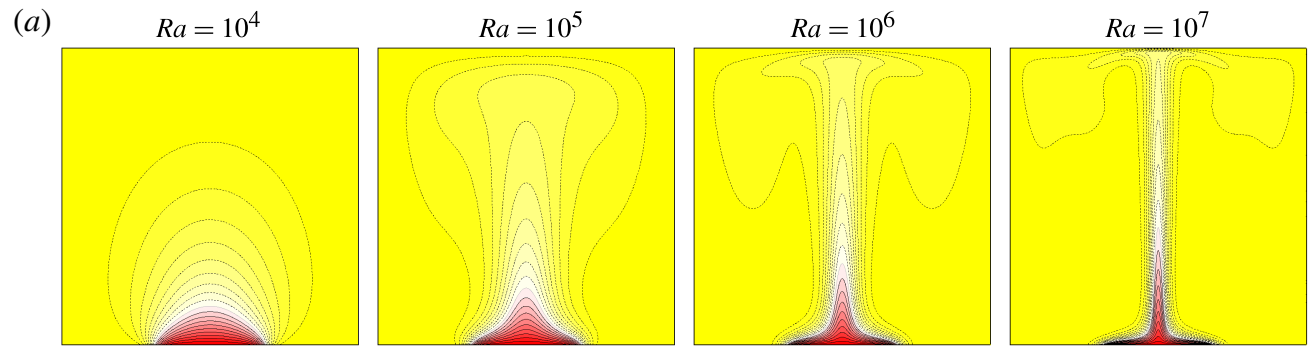

(b)
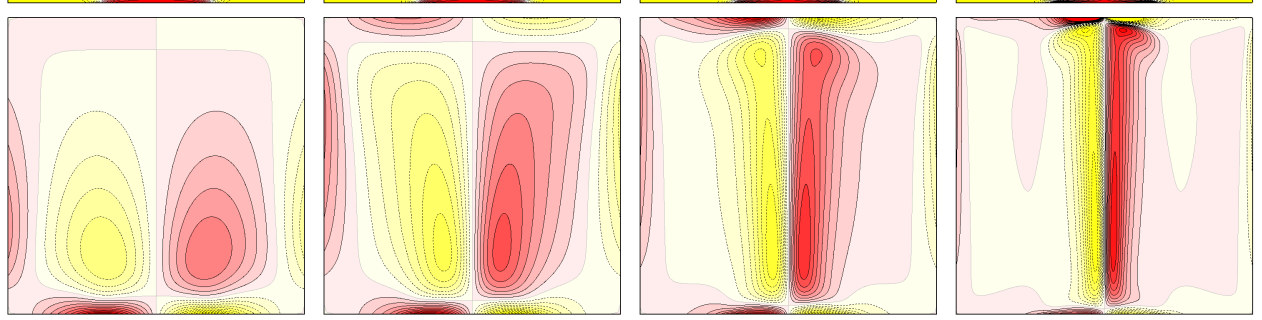

(c)
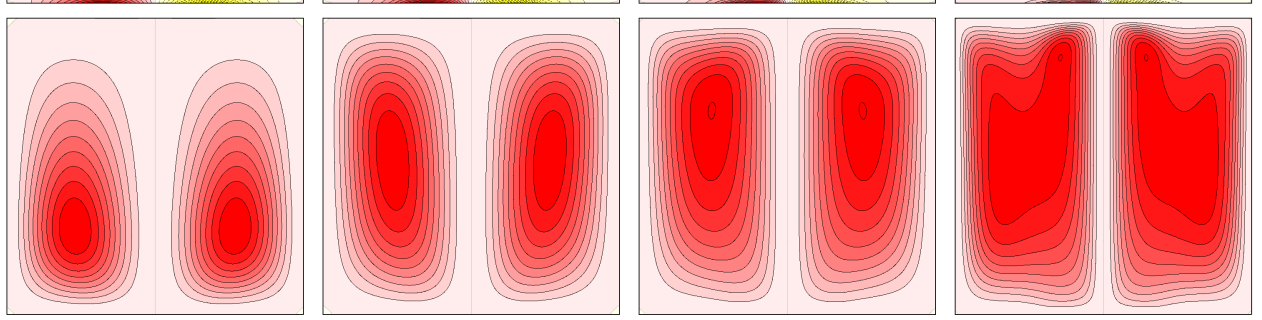

FIGURE 3. (Colour online) (a) Isotherms, (b) azimuthal vorticity contours and (c) streamlines of the basic state for $\gamma=0.5, \sigma=7, r_{w}=r_{d}=0.125$ and $R a$ as indicated; there are ten positive (solid lines and dark grey, red online) and ten negative (dotted lines and light grey, yellow online) levels with $T \in[-0.5,0.5]$ for all $R a, \eta \in[-180,180]$ and $\psi \in[-0.375,0.375]$ for $R a=10^{4}, \eta \in[-1500,1500]$ and $\psi \in[-3.5,3.5]$ for $R a=10^{5}$, $\eta \in[-8500,8500]$ and $\psi \in[-10.5,10.5]$ for $R a=10^{6}$, and $\eta \in\left[-5 \times 10^{4}, 5 \times 10^{4}\right]$ and $\psi \in[-14.8,14.8]$ for $R a=10^{7}$.

gradient for any non-zero $R a$, and this non-zero radial temperature gradient locally produces azimuthal vorticity; see (2.8). This vorticity drives a meridional circulation drawing fluid radially inward above the heated portion of the lower endwall and upward along the axis. The vertical extent of this axial upflow is limited by the diffusion of both temperature and vorticity. For very small values of $R a$, this advection is weak and the transport of heat is conduction dominated. Convection begins to dominate over conduction by about $R a=10^{4}$. Figure 3 shows isotherms, azimuthal vorticity contours and streamlines of the basic state at $\gamma=0.5, \sigma=7$, and $r_{w}=r_{d}=0.125$ for $R a \in\left[10^{4}, 10^{7}\right]$, illustrating the development of the flow as convection takes over from conduction as the dominant mechanism.

As $R a$ is increased, the meridional flow is intensified into a plume, which is steady, axisymmetric and purely poloidal, i.e. the only non-zero component of vorticity is the azimuthal component $\eta$. The plume can be characterized in terms of $\eta$ : using the radius, $r_{\max }$, at which $\eta$ is a local maximum near the source, $\eta_{\max }$, as a scale of its width, the height of this local maximum, $z_{\max }$, as a measure of its vertical extent, and the value of $\eta_{\max }$ as a measure of its strength. Another quantity characterizing the plume is the maximum vertical velocity along the axis, $w_{\max }$. Figure 4 shows 

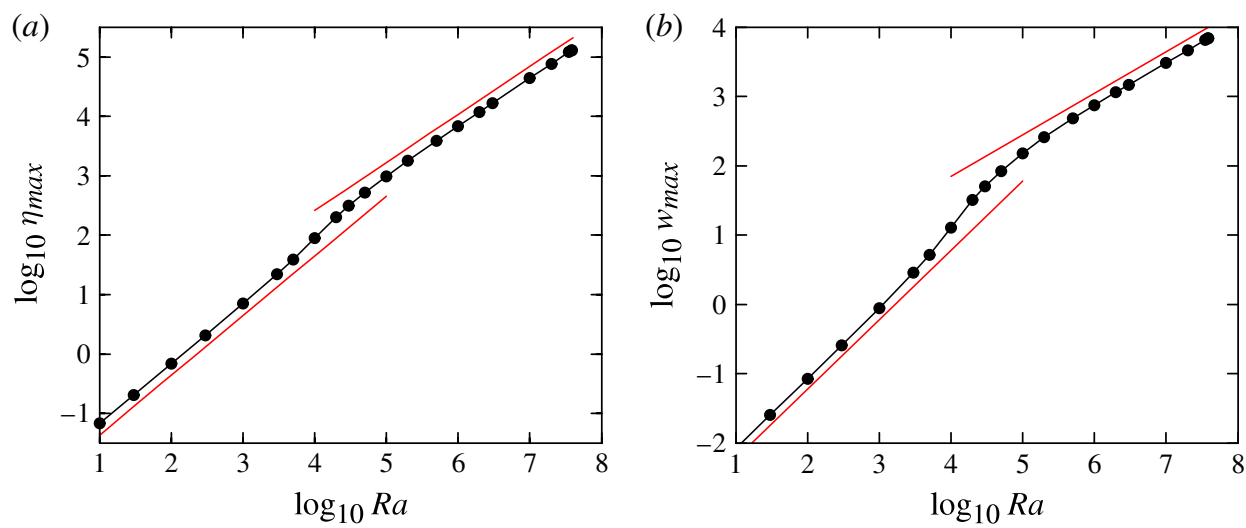

FIgURE 4. (Colour online) Variations of $(a) \eta_{\max }$ and $(b) w_{\max }$ with $R a$ for $\gamma=0.5, \sigma=7$ and $r_{w}=r_{d}=0.125$, plotted on a $\log -\log$ scale. The grey (red online) curves are straight line fits to the low- and high-Ra data.

the variations of $\eta_{\max }$ and $w_{\max }$ with $R a$ for $\gamma=0.5, \sigma=7, r_{w}=r_{d}=0.125$, plotted on a $\log -\log$ scale. It also includes fits for the low- and high- $R a$ data which show that $\eta_{\max } \propto R a$ and $w_{\max } \propto R a$ for $R a \lesssim 10^{3.5}$, and $\eta_{\max } \propto R a^{0.81}$ and $w_{\max } \propto R a^{0.6}$ for $R a \gtrsim 10^{5.5}$. These ranges of $R a$ may be termed the conduction-dominated and convection-dominated regimes, and the range in between corresponds to the transition.

A transition between conduction- and convection-dominated behaviour was also noted by Torrance \& Rockett (1969) in a similar range of $R a$ (they used a Grashof number $G r=R a / \sigma$ ), where their Prandtl number was $\sigma=0.7$ (we use $\sigma=7$ ), their aspect ratio was $\gamma=1$ (we use $\gamma=0.5$ ) and their heater radius was 0.1 (we use 0.25). We can directly compare the $R a$-scaling of $w_{\max }$ with Torrance \& Rockett (1969). They found that $w_{\max } \propto R a^{0.5}$, while we have a slightly larger value of the exponent. Note that they could only compute with a very coarse grid, and so used a highly diffusive finite-difference scheme so that the numerical viscosities would stabilize the numerical scheme. Hence, given the artificial viscosities they used, together with the differences in the parameters considered, the quantitative difference in the exponent is not surprising, but the qualitative behaviour is consistent.

In the conduction-dominated regime, the radial and axial locations of $\eta_{\max }$ remain approximately constant with respect to variations in $R a$, as can be seen from figure 5 . This is most probably due to the finite radial extent of the heated portion of the bottom, with $r_{\max }$ being a little smaller. As the advection strength increases with $R a$, $r_{\max }$ is reduced and $z_{\max }$ increases. The change in $z_{\max }$ is non-uniform in the transition regime as there is a competition between pinning the vorticity locally to the edge of the heater and advecting it away in the developing plume.

Note that the use of the streamfunction, $\psi$, is not particularly useful in this problem. It does not give an accurate impression of the development of an intense axial plume (see figure 3). This is because in order to extract the axial velocity, one needs the radial gradients in the streamfunction divided by the local radius. As such, when comparing radial gradients in $\psi$ we see that they are comparable near the axis and near the sidewall, but near the axis $r \approx 0$ and near the sidewall $r \approx 1$, so the axial velocity is actually much stronger near the axis and this is not conveyed well by the streamline plots. When the flow becomes unsteady (see $\S 4$ ), the unsteady streamlines are not good indicators of the flow (Hama 1962), and when the flow becomes 

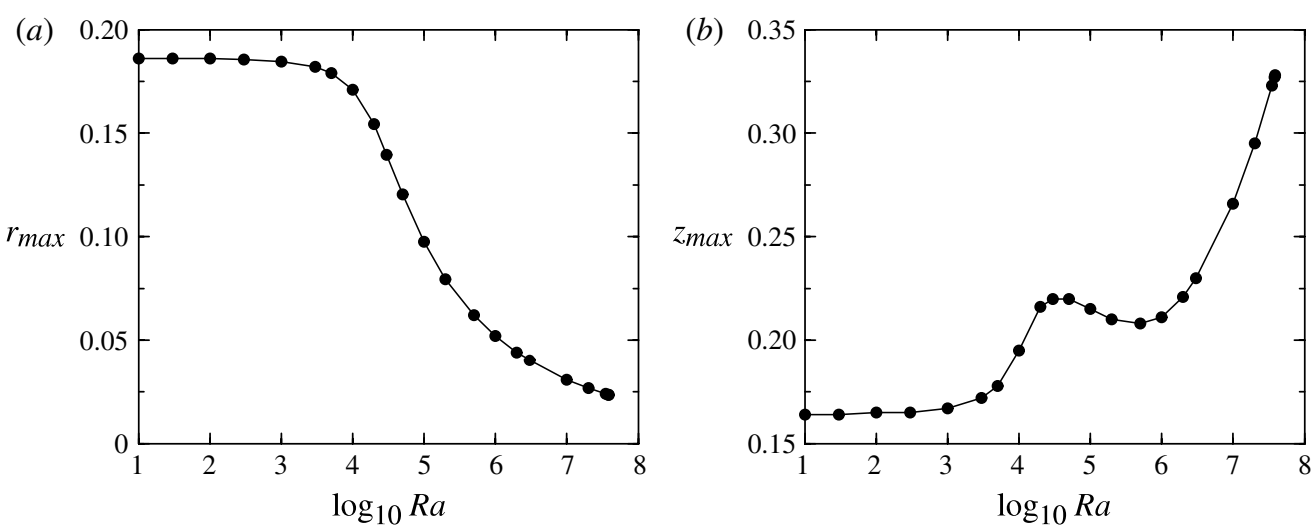

FIGURE 5. Variations of (a) $r_{\max }$ and (b) $z_{\max }$ with $R a$ for $\gamma=0.5, \sigma=7$ and $r_{w}=r_{d}=0.125$.

three-dimensional (see $\S 5$ ), the streamfunction is no longer defined. As such, we shall not present streamline plots henceforth.

Figures 6 and 7, together with figure 3, provide a summary of the effects of varying the geometry of the problem. Keeping $R a$ and $\sigma$ fixed, we can consider varying the aspect ratio $\gamma$ of the cylinder and the width of the heater $r_{w}$. If we keep the width constant at $r_{w}=0.125$, we see that the thermal and vortical structure of the plume is not affected very much. Compare figure $6(c, f)$ with figure $7(a, b)$ (all at $R a=10^{7}$ ), with the $R a=10^{7}$ isotherms and azimuthal vorticity contours in figure 3 . The difference between these three solutions basically corresponds to placing different sized radial shrouds about the same heater with the top at the same distance from the heater. The differences are primarily in the meridional circulation down the sidewall; for the smallest shroud radius, $\gamma=0.25$, the meridional circulation is strongest, but for increasing $\gamma$ the effects of the shroud are much reduced. Keeping everything the same and reducing the size of the heater diminishes the strength of the plume (see figure 6). Reducing the heater width and increasing $R a$ while keeping $\gamma$ constant effectively reduces the confinement effect of the top endwall. In order to approach the limit of a radially unbounded point-source heater requires $\gamma \rightarrow \infty$ and $r_{w} \rightarrow 0$ with $R a \rightarrow \infty$, but this is not practical numerically. A reasonable compromise is to use $\gamma=0.5$ and $r_{w}=0.125$ (see figure 3), for which the results are qualitatively insensitive to reasonable variations in $\gamma$ and $r_{w}$.

The structure of the laminar plume resembles the typical plume structure observed in analytical self-similar plume models (Pham et al. 2005; Plourde et al. 2008), and also exhibits the necking phenomenon (Kaye \& Hunt 2010). Although these selfsimilar models aim to describe turbulent plumes, they also share many characteristics with the laminar plumes in our case. Figure $8(a, b)$ shows the temperature and vertical velocity profile along the axis of the plume, for the solution at $\gamma=0.5, \sigma=7$, $r_{w}=r_{d}=0.125$ and $R a=10^{7}$ shown in the last column of figure 3 . We observe that the vertical velocity is constant except close to the hot spot, where the plume is formed due to the baroclinic term in (2.8), and also close to the top endwall, where the plume collides with the top wall and the velocity rapidly goes to zero. The temperature inside the plume decreases with $z$, reflecting the diffusion of the plume's heat to the isothermal ambient. The radial profile of temperature at a given height resembles a Gaussian, so we have used the distance between the axis and the inflection point of the 
(a)

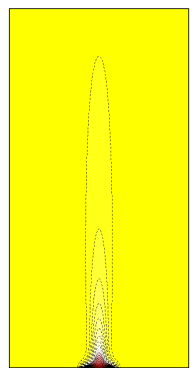

(b)

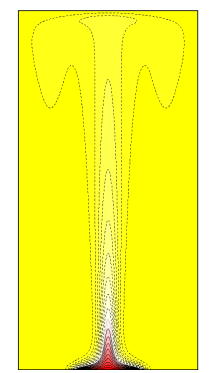

(c)

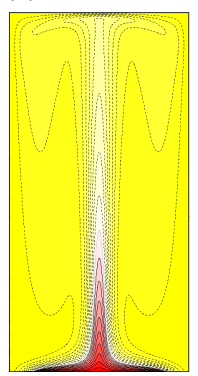

$(d)$

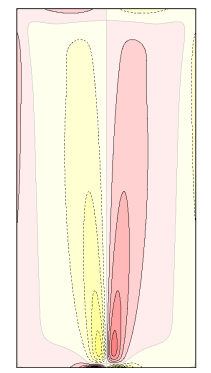

$(e)$

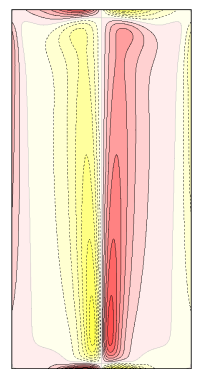

$(f)$

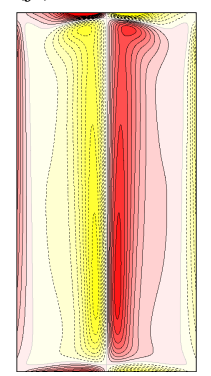

FIGURE 6. (Colour online) $(a-c)$ Isotherms and $(d-f)$ azimuthal vorticity contours of the basic state at $R a=10^{7}, \gamma=0.25, \sigma=7$; and $(a, d) r_{w}=r_{d}=1 / 32,(b, e) 1 / 16$ and $(c, f) 0.125$. There are ten positive (solid lines and dark grey, red online) and ten negative (dotted lines and light grey, yellow online) levels with $T \in[-0.5,0.5]$ and $\eta \in\left[-5 \times 10^{4}, 5 \times 10^{4}\right]$.

(a)

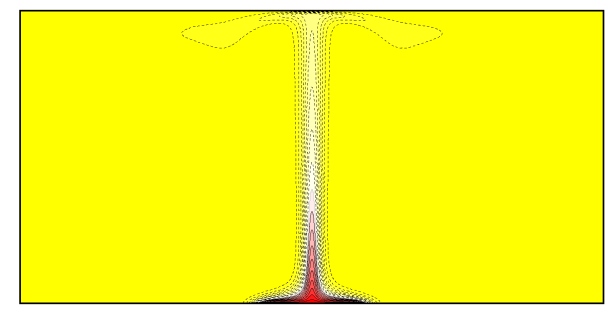

(b)

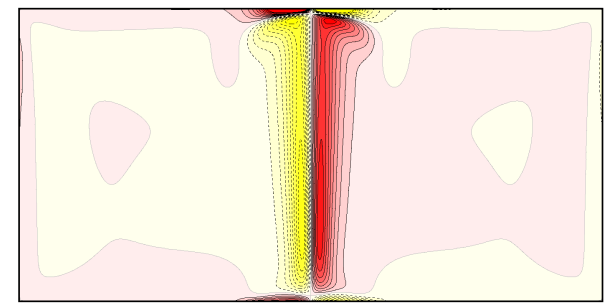

FIgURE 7. (Colour online) (a) Isotherms and $(b)$ azimuthal vorticity contours of the basic state at $R a=10^{7}, \gamma=1.0, \sigma=7$ and $r_{w}=r_{d}=0.125$; there are ten positive (solid lines and dark grey, red online) and ten negative (dotted lines and light grey, yellow online) levels with $T \in[-0.5,0.5]$ and $\eta \in\left[-5 \times 10^{4}, 5 \times 10^{4}\right]$.

radial temperature profile as a measure of the plume's radius. Figure $8(c)$ shows the resulting plume structure, with a well-defined neck at about $z=0.18$, or 1.44 times the hot-spot radius. Away from the endwalls, the plume has a conical shape, with a virtual origin well below the hot spot, located at about $z=-1.76$ (approximately 14 hot-spot radii below the bottom), and with a small cone half-angle $\alpha=0.6^{\circ}$. While these features are qualitatively similar to experimentally observed turbulent plumes (e.g. Colomer, Boubnov \& Fernando 1999; Fannelop \& Webber 2003; Pham et al. 2005; Kaye \& Hunt 2010), there are significant quantitative differences that are probably due to our plume being steady and laminar.

\section{Axisymmetric Hopf bifurcation: 'puffing' plume}

Now consider increasing $R a$ while keeping $\gamma=0.5, \sigma=7$ and $r_{w}=r_{d}=0.125$ fixed. At $R a=10^{7}$, we have a well-developed steady axisymmetric plume (figure 3). On further increasing $R a$, the flow undergoes a supercritical Hopf bifurcation at $R a \approx 3.854 \times 10^{7}$. The flow remains axisymmetric and poloidal (the only non-zero component of vorticity is $\eta$, the azimuthal component). Using the temperature on the axis at mid-height $T_{m}=T(r=0, z=0.5)$ as a measure of the flow state to characterize the limit cycle, we define the limit-cycle amplitude as $\Delta T_{m}$, equal to 

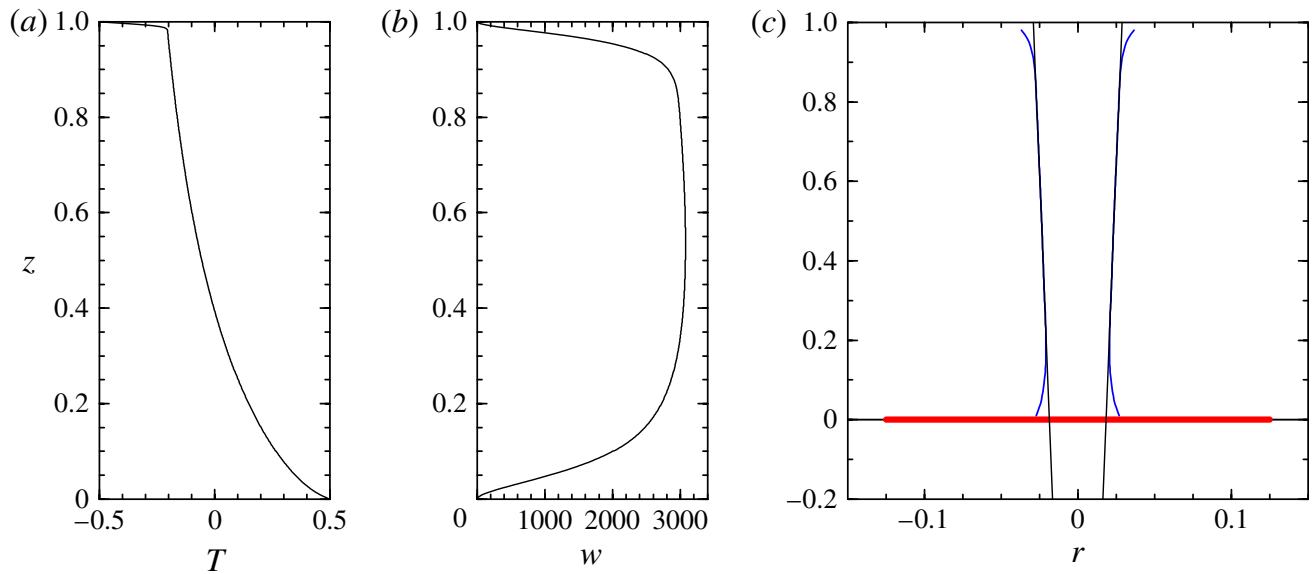

FIgURE 8. (Colour online) Axial profiles of $(a)$ temperature and $(b)$ vertical velocity of the laminar plume for $\gamma=0.5, \sigma=7, r_{w}=r_{d}=0.125$ and $R a=10^{7}$ (the plume is depicted in the last column of figure 3). (c) Loci of the inflection points of the plume's radial temperature profile, showing the plume structure. The thick line indicates the extent of the heater.
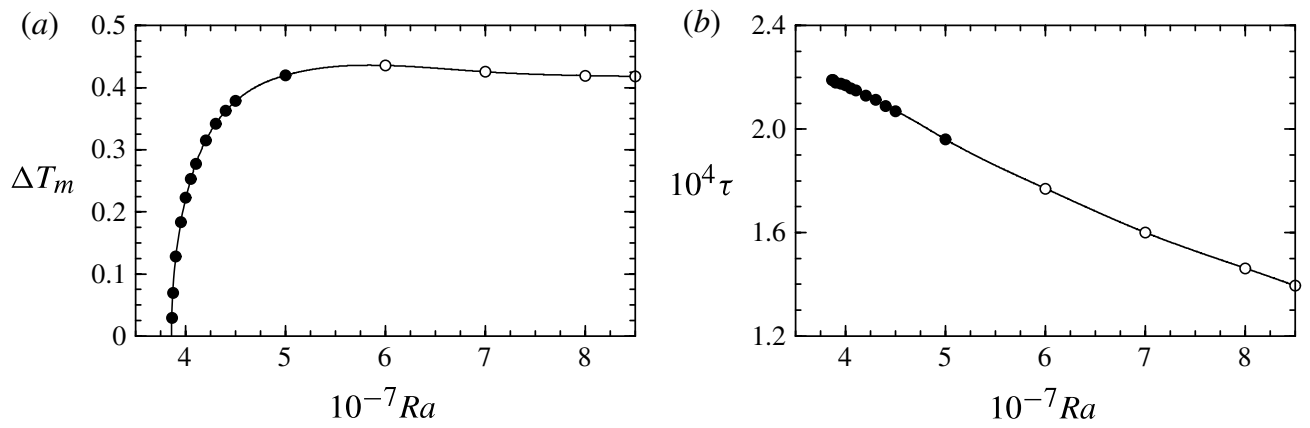

FIgURE 9. (a) Amplitude and (b) period of the $O(2)$-symmetric limit-cycle solution as a function of $R a$. The open symbols correspond to states computed in the $O(2)$-symmetric subspace which are unstable to an $m=1$ azimuthal mode for $R a>5.3 \times 10^{7}$.

the peak-to-peak difference in $T_{m}$ over one period. Figure 9 shows the amplitude $\Delta T_{m}$ and the corresponding oscillation period $\tau$ as a function of $R a$. The Hopf bifurcation is supercritical, but the amplitude rises very sharply with small increases in $R a$, suggesting that it is close to being a subcritical Hopf bifurcation. This is consistent with many reports of plumes in various experimental and numerical settings where on the experimental side it is noted that transition from steady to unsteady flow is catastrophic, and numerically, noise needs to be introduced in order to force an unsteady flow.

Figure 10 shows instantaneous isotherms and azimuthal vorticity contours of the 'puffing' state at $R a=5 \times 10^{7}$ over a puffing period of $\tau \approx 2 \times 10^{-4}$, and the online version includes movies of these (available online at http://dx.doi.org/10.1017/jfm. 2013.537). Prior to the Hopf bifurcation responsible for the puffing state, the radial temperature gradient in the neighbourhood of the heat source leads to radial inflow and 
(a)

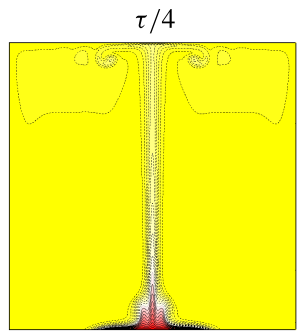

(b)

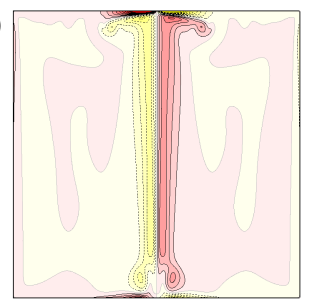

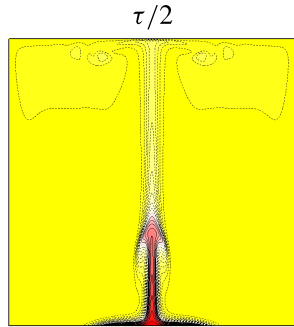

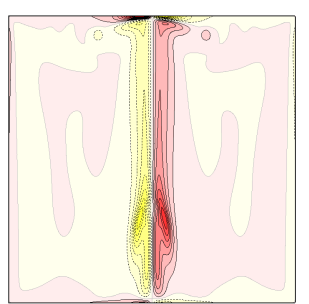

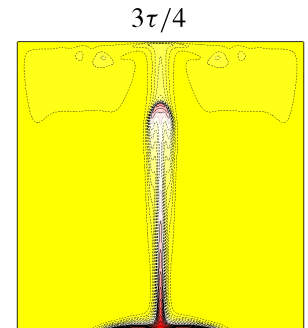

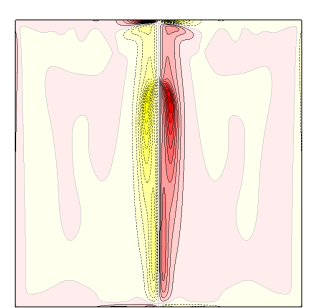

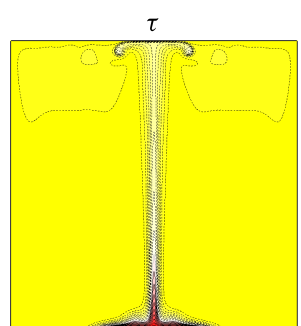

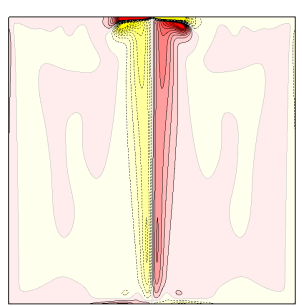

FIGURE 10. (Colour online) (a) Isotherms and $(b)$ azimuthal vorticity at four phases over one period of the axisymmetric periodic state at $R a=5 \times 10^{7}$. There are ten positive (solid lines and dark grey, red online) and ten negative (dotted lines and light grey, yellow online) levels with $T \in[-0.5,0.5]$ and $\eta \in\left[-3 \times 10^{5}, 3 \times 10^{5}\right]$. Movies are available online at http://dx.doi. org/10.1017/jfm.2013.537.

the formation of the plume via the baroclinic torque; see (2.8). As $R a$ is increased, the radial inflow (driven by the baroclinic torque) at the base becomes too fast for the upward advection (driven by the buoyancy) in the plume, and a toroidal vortex ring is formed at the heater with the temperature in the core of the ring hotter than the immediate surroundings, producing a buoyancy anomaly. As the ring intensifies, its induced circulation together with the additional buoyancy from the core anomaly propels it axially upward faster than the flow in the core of the plume. It advects the relatively hot fluid with it, and so as it leaves the heater, the radial temperature gradient is reduced. Then, over time, the radial temperature gradient is re-established from the heater and the whole process is repeated, leading to the puffing phenomenon. This has been observed in experiments (Jaluria \& Gebhart 1975; Maxworthy 1999) and in simulations (Jiang \& Luo 2000) in related localized heating problems.

\section{The three-dimensional 'flickering' plume}

The axisymmetric 'puffing' plume becomes unstable to three-dimensional disturbances at about $R a=5.4 \times 10^{7}$. The most amplified Fourier mode corresponds to azimuthal wavenumber $m=1$, and corresponds to a tilting of the puff (see figure 14 below). Figure 11 shows time series of $E_{1}$, the kinetic energy associated with the azimuthal wavenumber $m=1$ component of the velocity field, for various $R a$. For $R a=5 \times 10^{7}$, a small non-axisymmetric perturbation was added to the puffing solution and allowed to evolve. The figure shows that $E_{1}$ decays exponentially by about one order of magnitude for every $1 \%$ of a viscous time (the other $E_{m}$, $m>1$, decay even faster). Repeating this exercise for $R a=5.3 \times 10^{7}$ has similar results, but the exponential decay takes much longer to set in and the rate is much slower. For $R a=5.5 \times 10^{7}$, the three-dimensional perturbations do not decay, and instead, $E_{1}$ saturates to a mean value of order $10^{4}$. Figure 12 shows time-averaged 


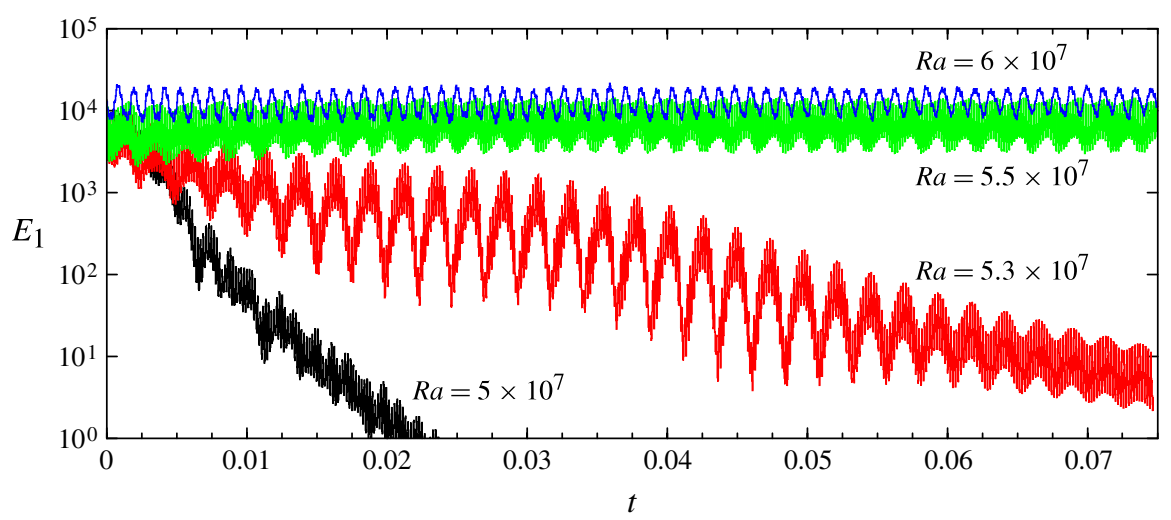

FIgURE 11. (Colour online) Time series of $E_{1}$ for $R a$ as indicated.

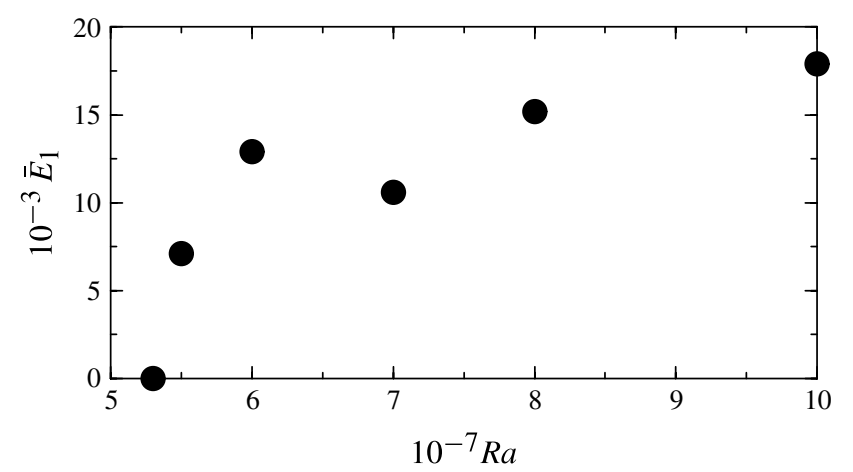

FIGURE 12. Variation of the time-averaged $m=1$ modal energy, $\bar{E}_{1}$, with $R a$.

$E_{1}$ versus $R a$ for several $R a$, and is indicative of a supercritical bifurcation breaking the axisymmetry, and since the time series of $E_{1}$ shows a fast oscillation with a slow modulation, this bifurcation is a secondary Hopf bifurcation that introduces the new (modulation) frequency.

The fast oscillations in the $E_{1}$ time series correspond to the axisymmetric puffing. The $E_{0}$ time series, corresponding to the kinetic energy of the axisymmetric component of the flow, shows the same characteristic behaviour. Figure 13 presents power spectral densities (PSD) of the $E_{0}$ time series for various $R a$. The $R a=5 \times 10^{7}$ case is a purely axisymmetric puffing state, and its PSD consists of a peak at the puffing frequency $\omega=2 \pi / \tau$, where $\tau$ is the puffing period shown in figure $9(b)$, together with several harmonics. For $R a=5.5 \times 10^{7}$, the PSD also consists of a peak at the puffing frequency (it is a little higher since $\tau$ decreases with $R a$, as shown in figure $9 b$ ), its harmonics, plus side bands. The side-band frequency (the peak at the smallest frequency) is $\sim 1 / 10$ of the puffing frequency, which corresponds to the modulation frequency shown in the $E_{1}$ time series in figure 11 . The two frequencies are incommensurate, and the flow is quasi-periodic. At higher $R a$, the modulation is stronger, but the underlying puffing is still dominant. This is clearly seen in the PSD of both the $R a=6 \times 10^{7}$ and $R a=7 \times 10^{7}$ cases. These are no longer quasi-periodic 

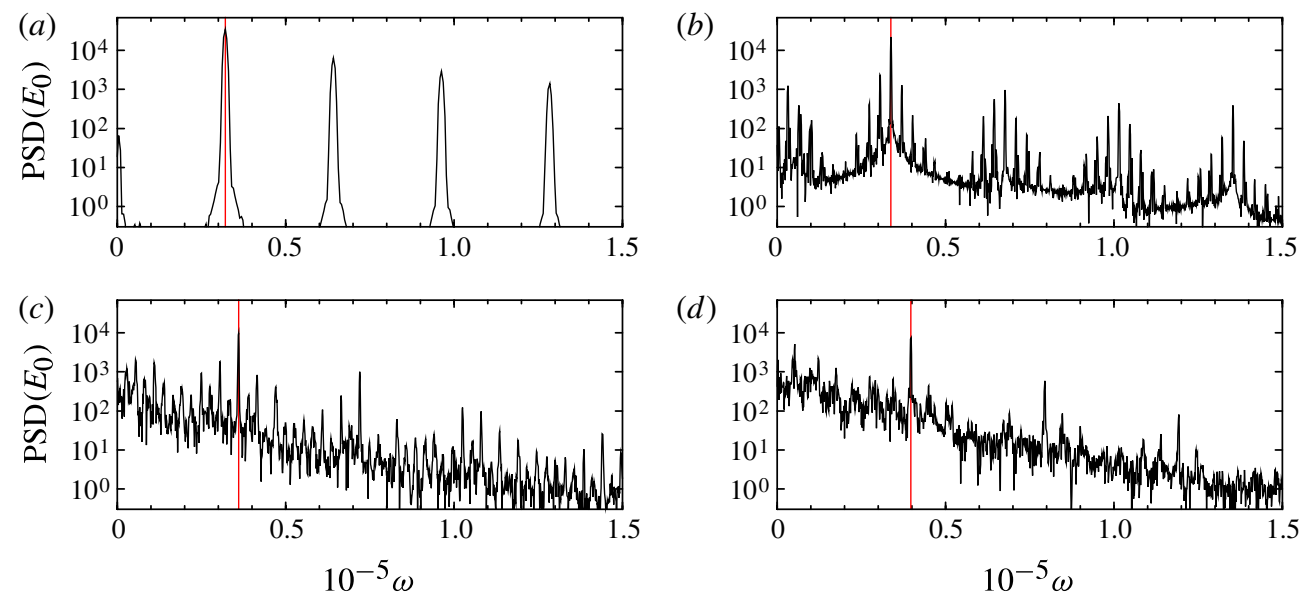

FIgURE 13. (Colour online) Power spectral density of $E_{0}$ for $(a) R a=5 \times 10^{7}$, (b) $R a=5.5 \times 10^{7}$, (c) $R a=6 \times 10^{7}$ and $(d) R a=7 \times 10^{7}$ The grey (red online) vertical line indicates the 'puffing' frequency.

and additional symmetry-breaking bifurcations have taken place (these and higher- $R a$ states are described below).

Figure 14 gives an indication of what the modulation corresponds to in the flow at $R a=5.5 \times 10^{7}$. It shows isotherms and contours of $\eta$ in a meridional plane which are similar to those for the axisymmetric puffing state at $R a=5 \times 10^{7}$, except that the flow is no longer axisymmetric. The concentrated pulse becomes tilted (azimuthal wavenumber $m=1$ ) as it propagates up the plume at about the same puffing frequency as in the underlying axisymmetric puffing state. The tilted puff results in the plume core deviating off axis and the two interact in what appears to be a wobbling of the puff, leading to a 'flickering' plume. However, it is quite different to the flickering candle flames observed by Maxworthy (1999); those had a helical mode whereas we have a meandering tilt in a fixed meridional plane. This is more clearly seen in the three-dimensional isosurfaces of $\eta$ and $\zeta$, the azimuthal and axial components of vorticity, shown in figure 15. From these (and the accompanying online movies) it is more readily seen that the puff emerges from the heater as an axisymmetric puff that becomes tilted as it rises. The axial vorticity primarily results from the azimuthal vorticity associated with the axisymmetric puff being tilted into the axial direction. The meridional plane in which the puff is tilted is fixed is time, and the tilted puff and plume core wobble and meander in this fixed plane. Although this meridional plane is readily identified and steady, i.e. it does not precess, the flow is not reflection-symmetric with respect to this plane. That the reflection symmetry is only approximately fulfilled can been seen by comparing the positive and negative axial vorticity isosurfaces in figure 15 . So, for $R a=5.5 \times 10^{7}$, not only is the $S O$ (2) part of the $O(2)$ symmetry broken (by the $m=1$ tilt), but so is the $Z_{2}$ part of the $O(2)$ symmetry (the tilt not being reflection-symmetric across the tilt plane). This $Z_{2}$ symmetry breaking gives rise to the slow modulation in the puff cycles, evident in the PSD shown in figure 13. All of this takes place over a small range in $R a \in\left(5.3 \times 10^{7}, 5.5 \times 10^{7}\right)$. We have not attempted to resolve this as it is computationally very intensive. This solution is very close to the modulated standing wave predicted by normal-form theory, so the most likely scenario is a bifurcation 

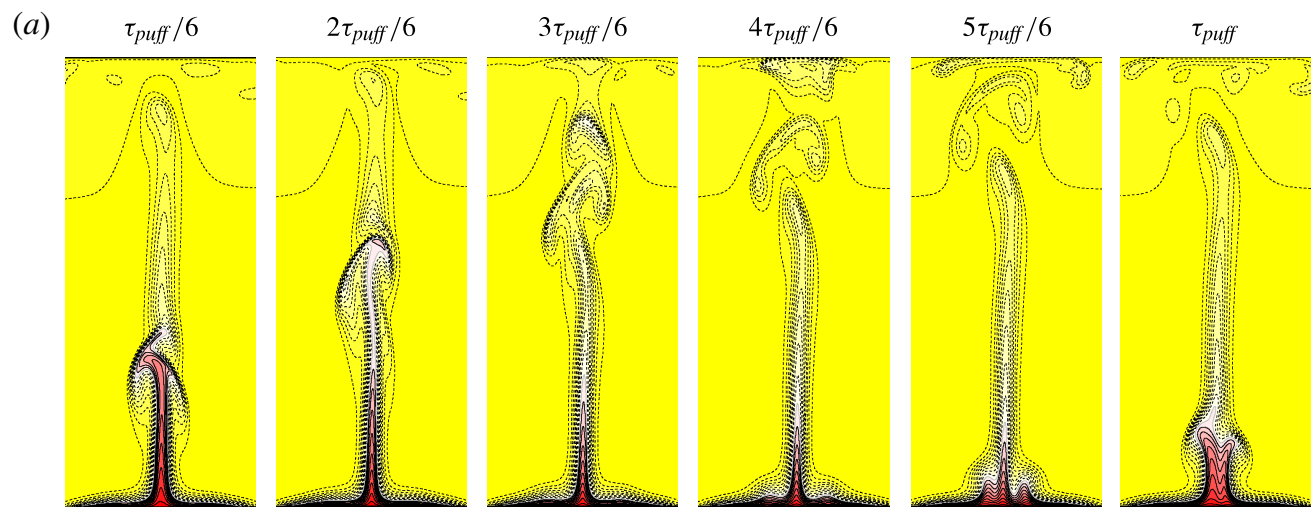

(b)
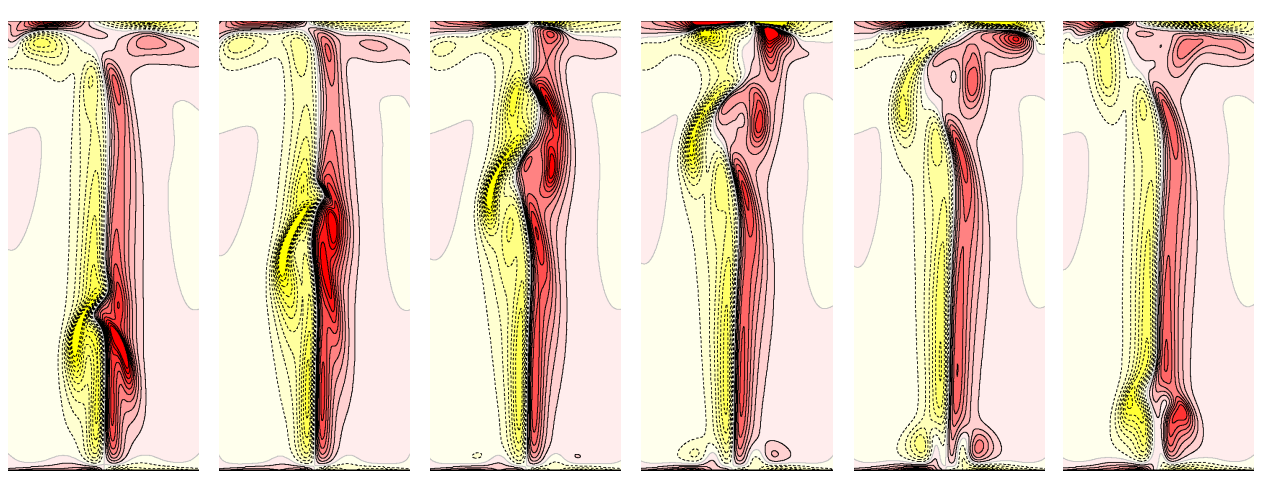

FIGURE 14. (Colour online) ( $a$ ) Isotherms and $(b) \eta$-contours of the plume at $R a=5.5 \times 10^{7}$, over one puff cycle $\tau_{\text {puff }} \approx 1.85 \times 10^{-4}$. The images have been cropped to show only the central plume region. There are ten positive (solid lines and dark grey, red online) and ten negative (dotted lines and light grey, yellow online) levels with $T \in[-0.5,0.5]$ and $\eta \in\left[-2 \times 10^{5}, 2 \times 10^{5}\right]$.

to a modulated standing wave which is reflection-symmetric, closely followed by a secondary bifurcation breaking the reflection symmetry. It could also be possible that we are close in parameter space to a degenerate bifurcation, and we are observing a mixed mode that has broken all symmetries, as discussed at the end of $\S 2$. In either case, the end result is that by $R a=5.5 \times 10^{7}$ both symmetries are broken and the solution is quasi-periodic.

Although the orientation of the tilt plane is time-invariant, this orientation is arbitrary and determined by initial conditions, i.e. there is a whole circle of such flickering solutions parametrized by the azimuthal orientation of their meridional fixed plane. This is a consequence of breaking $O(2)$ symmetry, and has been observed experimentally in some plume studies. For example, Kimura \& Bejan (1983) conducted experiments with an isolated plume in air using smoke visualization, focusing on the symmetry breaking of the laminar plume. They report that the transitional plume 'meanders in a plane with a wavelength which scales with the plume diameter'. The plume is oriented in a meridional plane, and the angle of the plane is arbitrary and can be changed by 'tapping on the side of the box once'. They noted that this contradicts the assumption of Batchelor \& Gill (1962) that the primary instability mode of a free jet is helical. It is now appreciated that the analysis 
(a)

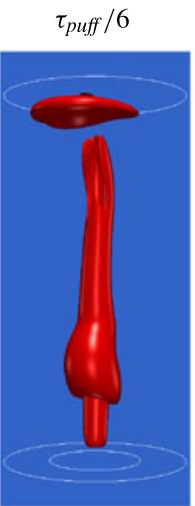

$$
2 \tau_{\text {puff }} / 6
$$

$3 \tau_{\text {puff }} / 6$
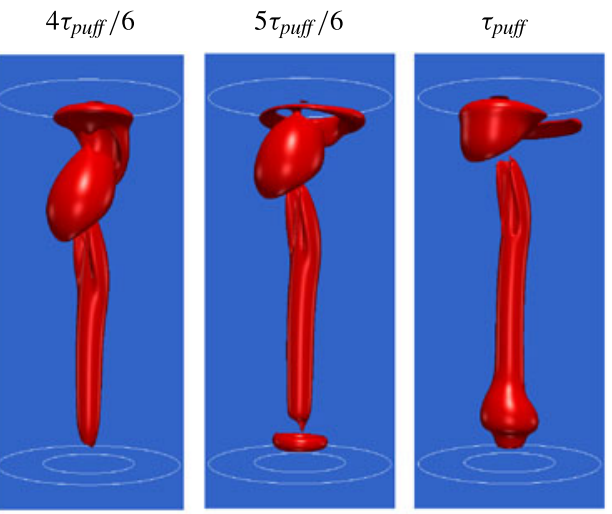

(b)
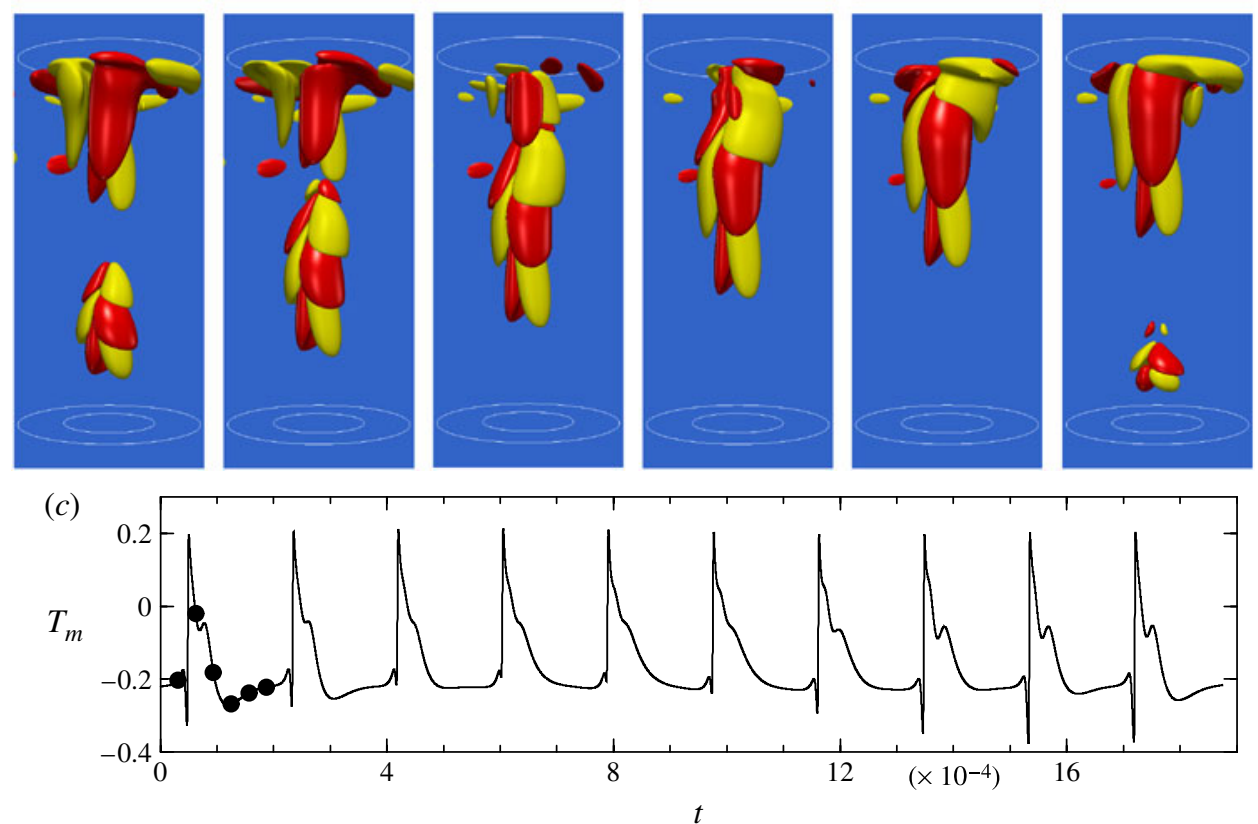

FIGURE 15. (Colour online) Isosurfaces of $(a)$ the azimuthal vorticity (level $\eta=8 \times 10^{4}$ ) and $(b)$ axial vorticity (levels $\zeta= \pm 5 \times 10^{3}$, red/dark grey is positive and yellow/light grey is negative) of the plume at $R a=5.5 \times 10^{7}$, over one puff cycle $\tau_{\text {puff }} \approx 1.85 \times 10^{-4}$. The images have been cropped to show only the central plume region. The symbols in the time series of $T_{m},(c)$, indicate the times corresponding to the snapshots.

of Batchelor \& Gill (1962) was incomplete as they only considered a rotating wave perturbation, which would be fine if the system were $S O(2)$ equivariant. However, being $O(2)$ equivariant, instability eigenmodes have multiplicity two and they should have included the complex conjugate as well, so that they would have considered rotating waves of left and right parity. As is also now well-known, $O(2)$ symmetry breaking results in either a rotating wave (the parity depending on initial conditions), or a standing wave (sum of the two conjugate rotating waves) (e.g. Crawford \& Knobloch 1991; Knobloch 1996). These types of incomplete stability analyses of $O(2)$ symmetry breaking are often made (e.g. Yang 1992; Hu \& Patankar 1995), leading to potentially incorrect conclusions. In our problem, the $O(2)$ symmetry breaking is not 
a Hopf bifurcation from a steady state, but rather a Hopf from an $O(2)$ equivariant limit cycle (the puffing solution). Nevertheless, the dynamical consequences are similar (Marques et al. 2004), in that the (modulated) standing wave and the rotating waves all bifurcate at the same critical point in parameter space, and if they are all supercritical then either the standing or the rotating wave is stable, the one with the largest amplitude being the stable one, otherwise neither is stable. In our problem, as in the experiments of Kimura \& Bejan (1983), the modulated standing wave is the resultant stable state.

This modulated standing wave (the flickering plume) exists on an invariant (by time evolution) two-torus, and generically (e.g. when the two frequencies are incommensurate), the flickering plume is dense on the torus. The individual solutions are not reflection-invariant as they have undergone a secondary bifurcation breaking reflection symmetry; however the symmetrically related solution lives on the same torus, as we have observed by time evolution of a reflected solution. Therefore the two-torus as a set is reflection-symmetric, although the individual solutions on it are not. Further, there is continuous family of these tori, corresponding to the different azimuthal orientations of the plume plane.

It is also interesting to note that the lack of reflection symmetry (the $Z_{2}$ symmetry breaking) is most prominent when the puff hits the top endwall, as is clearly seen in figure 15. For increasing $R a$, the lack of reflection symmetry extends further down. This suggests that the secondary bifurcation related to the breaking of the reflection symmetry is physically related to the collision of the thermal plume with the top endwall.

At larger $R a>10^{8}$, the character of the flickering plume changes, essentially via a torus-breakup bifurcation, and the flow is non-periodic. The flow intensity is much stronger, and the associated hydrodynamic mixing leads to the plume being nearly isothermal by half-height, but the flow intensity (all three components of vorticity) remains strong all the way up.

Figures 16 and 17 show the corresponding time series of the same variables as in figure 14, but for higher Rayleigh numbers, $7 \times 10^{7}$ and $3 \times 10^{8}$ respectively. Although the $R a=7 \times 10^{7}$ case in figure 16 looks very similar to the quasi-periodic solution at $R a=5.5 \times 10^{7}$ in figure 14, the Fourier spectrum (figure 13d) is more complex, with more than two frequencies and the presence of a broad band, a clear indication of the torus breakup. Here, a new dynamics has appeared: the (tilted) puff ring is emitted regularly from the bottom endwall, essentially at the puffing period. As well as the puff ring, at this higher $R a$ the central plume develops a hot spot that locally generates a smaller vortex ring. The two (tilted) rings play leap-frog, and in each puffing period their interaction is slightly different, leading to more complex dynamics, as illustrated in figure 16 and the accompanying supplementary movie. The time series of the temperature on the axis at mid-height $T_{m}$ in figure $16(d)$ is still fairly regular; the two peaks corresponding to the slow and fast puffs, and a weak modulation are evident. The power spectral density of the corresponding $E_{0}$ time series (figure $13 d$ ) clearly shows strong signals at the puffing frequency $\left(\approx 4 \times 10^{-6}\right)$ and the modulation frequency $\left(\approx 5 \times 10^{-7}\right)$.

On further increasing the Rayleigh number, the plume becomes chaotic. Figure 17 shows isotherms and azimuthal vorticity contours at $R a=3 \times 10^{8}$ and gives some indication of the spatial and temporal chaotic character of the solution. However, this chaotic solution still retains some semblance of the meridional plane that is temporally 
(a)

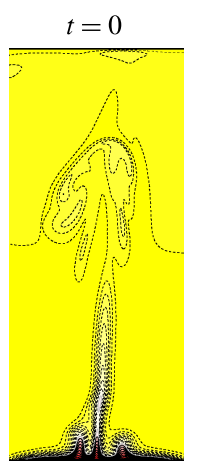

(b)

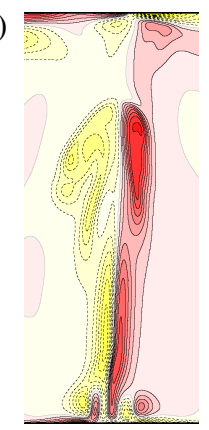

(c)

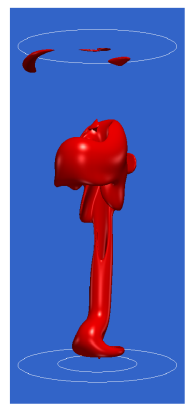

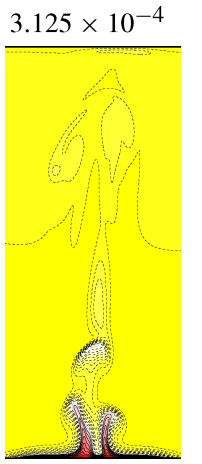
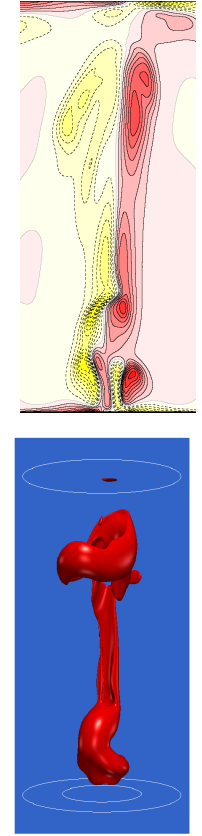
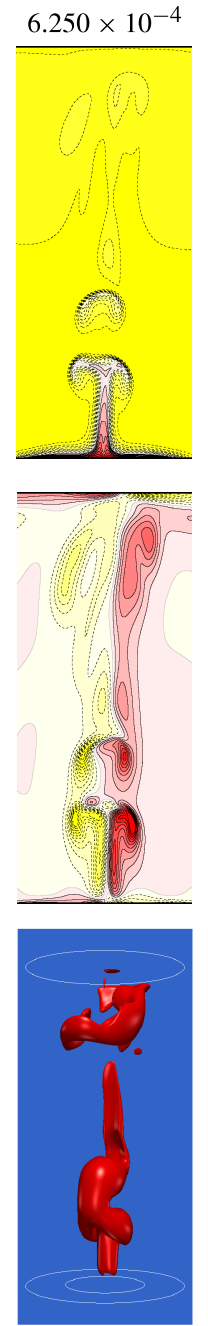
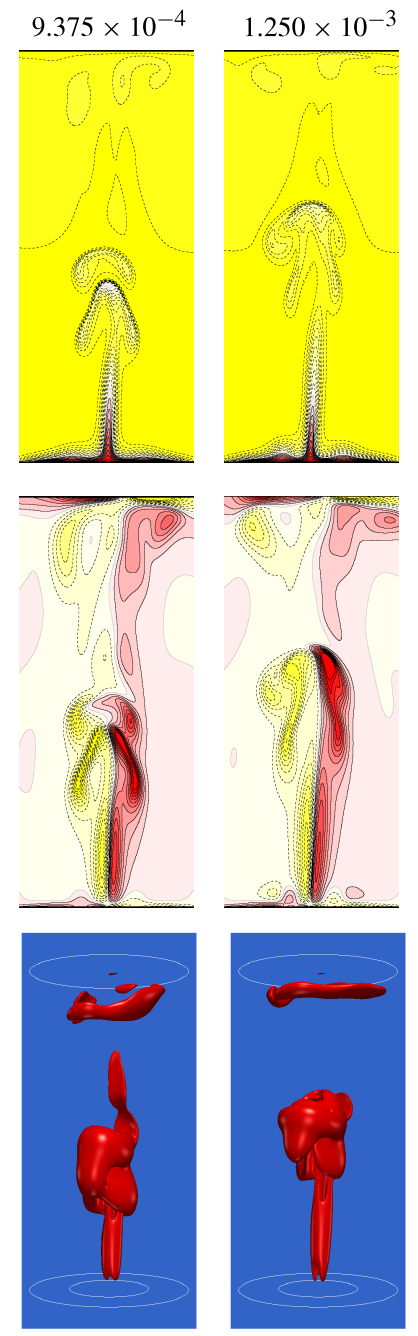
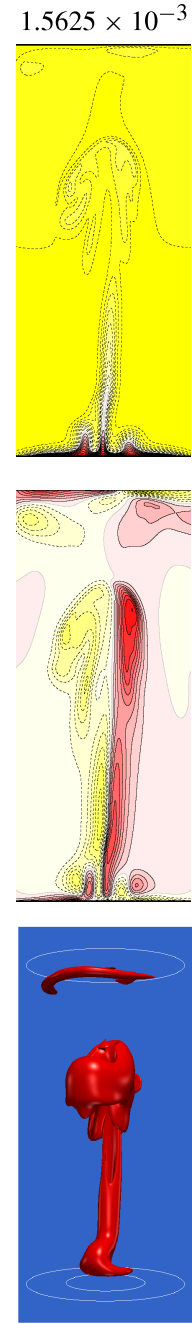

(d)

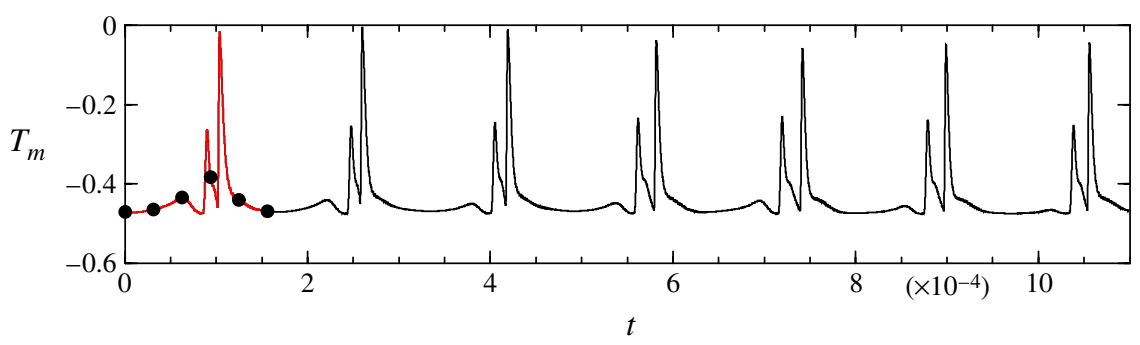

FIGURE 16. (Colour online) (a) Isotherms, (b) $\eta$-contours, and (c) $\eta$-isosurface (level $\eta=8 \times 10^{4}$ ) of the plume at $R a=7 \times 10^{7}$, over one puff cycle $\tau_{\text {puff }} \approx 1.75 \times 10^{-4}$. The images have been cropped to show only the central plume region. There are ten positive (solid lines and dark grey, red online) and ten negative (dotted lines and light grey, yellow online) contour levels with $T \in[-0.5,0.5]$ and $\eta \in\left[-2 \times 10^{5}, 2 \times 10^{5}\right]$. The time series of $T_{m},(d)$, indicates the times corresponding to the snapshots (symbols) and a movie over the segment with symbols is available online. 

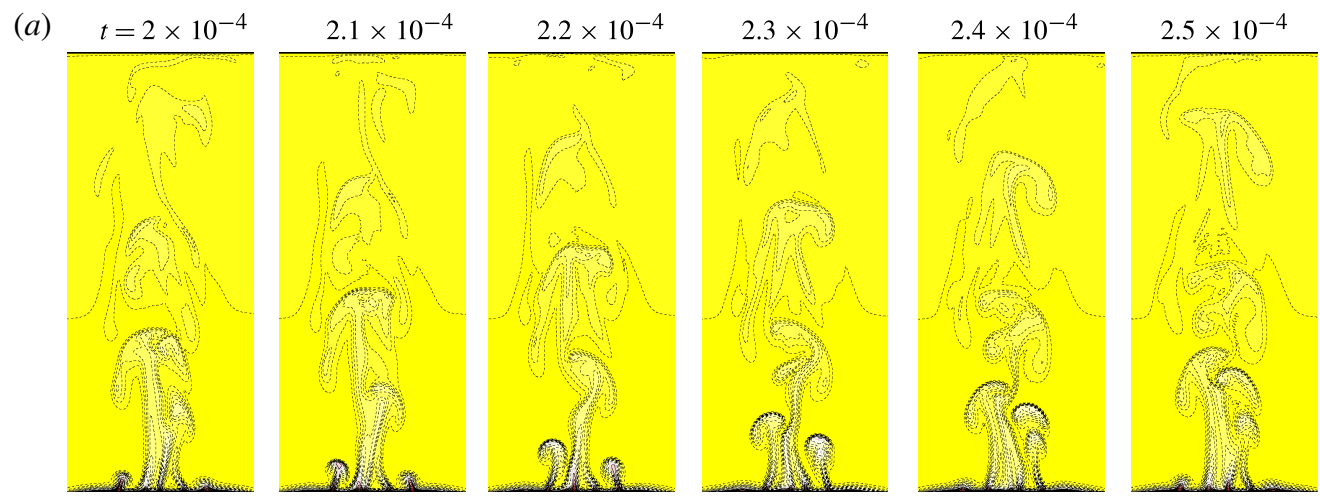

(b)
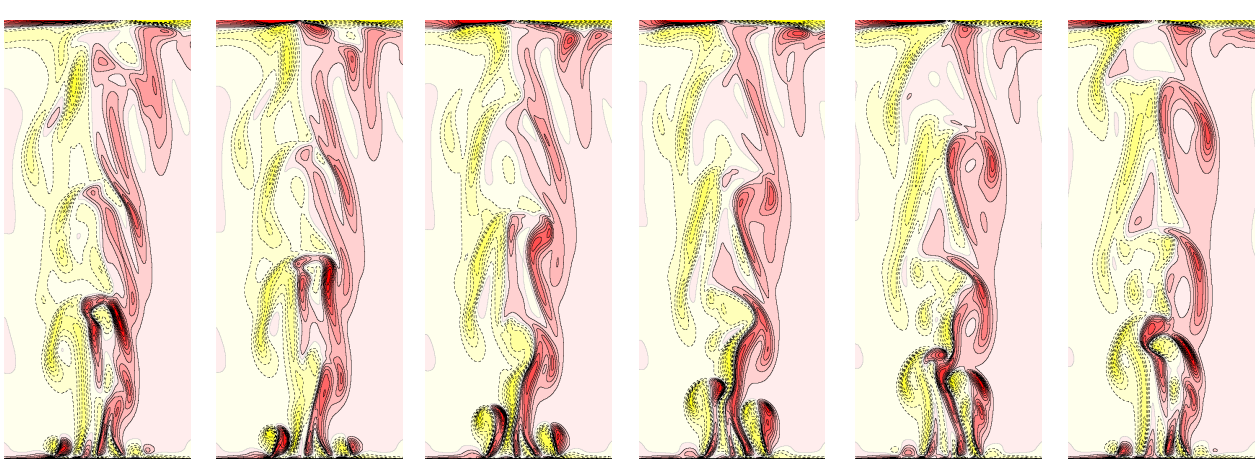

FIGURE 17. (Colour online) ( $a$ ) Isotherms and (b) $\eta$-contours of the plume at $R a=3 \times 10^{8}$, at times as indicated. The images have been cropped to show only the central plume region. There are ten positive (solid lines and dark grey, red online) and ten negative (dotted lines and light grey, yellow online) contour levels with $T \in[-0.5,0.5]$ and $\eta \in\left[-5 \times 10^{5}, 5 \times 10^{5}\right]$.

invariant, and where the plume undergoes its chaotic wobbling and flickering motion. This is more clearly evident in figures 18 and 19 which show isosurfaces of the azimuthal and vertical vorticity, respectively, over two puffing periods. The time series of $T_{m}$ in figure 18(b) further illustrates the temporal complexity of the solution. Even though this time series is rather erratic, the sequence of isosurface plots (and the accompanying online movie) indicate that there is still a good deal of flow organization. In particular, the lower portion of the plume is still quite periodic. The ring which is emitted from the heater every puff period is tilted by a large angle, and this tilting is responsible for the large component of vertical vorticity, but the plane in which it is tilted remains fixed as in the lower $R a$ cases, although the solution is clearly not symmetric about this plane, and much less so than for the lower- $R a$ cases. However, in the top half of the plume, the tilt plane is no longer coherent, and in fact when the puffs collide with the top endwall, the ensuing ceiling jet travels radially outward preferentially with a different azimuthal bias for each puff.

Apart from the sequence of symmetry breakings in the transition from laminar to turbulent plume, the transition is also characterized by the interactions between puffs. For the axisymmetric puffs, at $R a=5 \times 10^{7}$, we saw from figure 10 that they traverse the height of the cylinder in one puff period and there is no puff-puff interaction. At slightly higher $R a=5.5 \times 10^{7}$ (figure 14), the puffs take a little longer, $\sim 10 \%$, to 

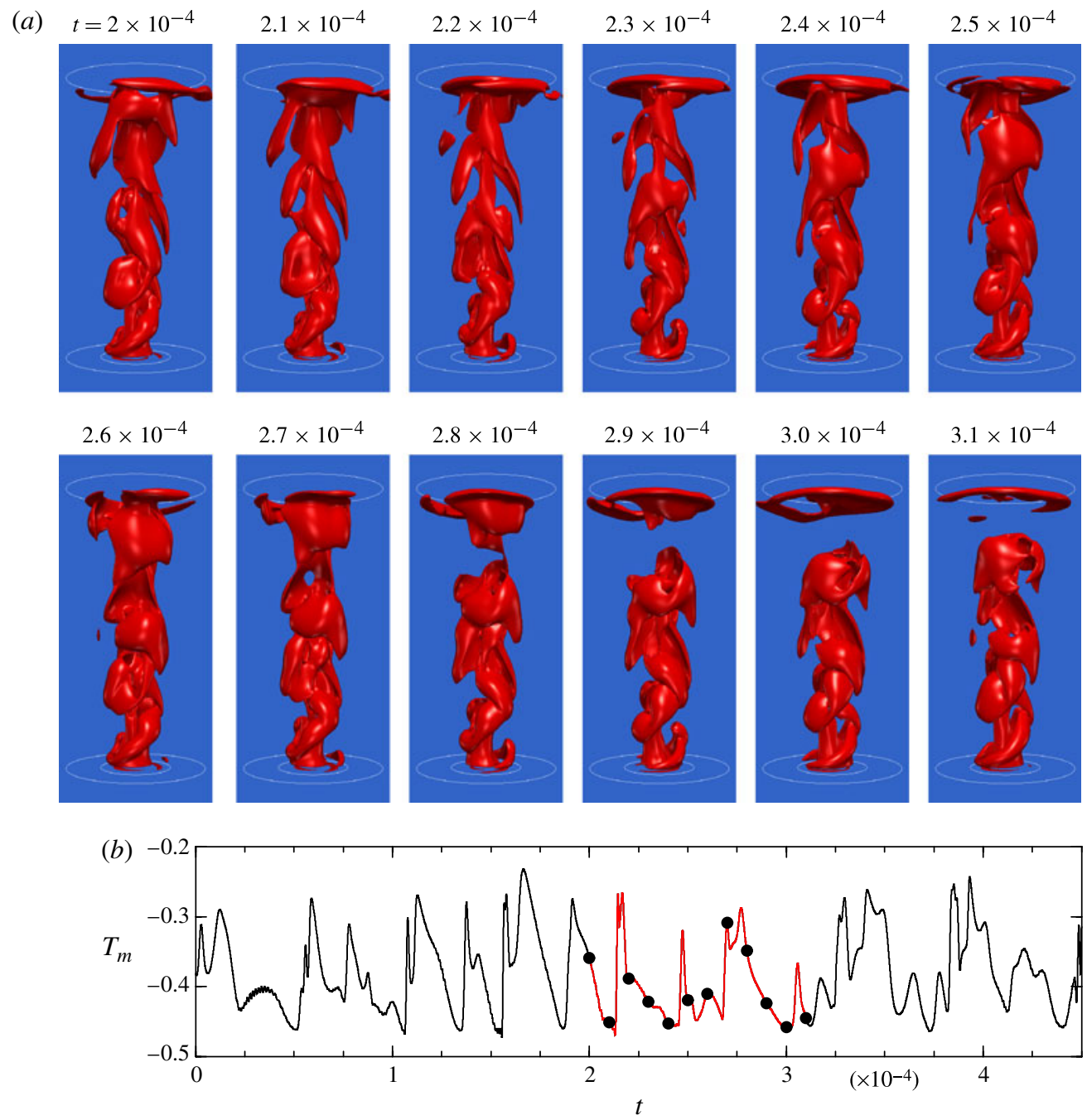

FIGURE 18. (Colour online) (a) Isosurfaces of azimuthal vorticity (level $\eta=1.4 \times 10^{5}$ ) of the plume at $R a=3 \times 10^{8}$, at times as indicated, covering two 'puff' periods. The images have been cropped to show only the central plume region. The time series of $T_{m},(b)$, indicates the times corresponding to the snapshots (symbols) and a movie over the segment with symbols is available online.

traverse the cylinder, and so there is a weak interaction between consecutively emitted puffs and the flow is weakly modulated quasi-periodic. By $R a=7 \times 10^{7}$, the tilted puffs take $\sim 1.5$ puff periods to traverse, and at $R a=3 \times 10^{8}$ they take over two puff periods to traverse. In these cases there is significant interaction between several puffs and this contributes to the fluid mixing and transition to turbulence.

At the higher $R a$, the 'localized' heat source behaves more like a finite distributed heat source in that instead of a single coherent plume emerging there are several plume-like eruptions that interact. The ratio of the boundary layer thickness to the radius of the heater is likely to be an important factor; this issue warrants further study. 

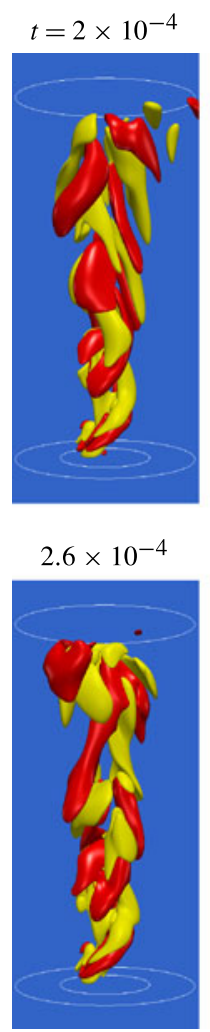
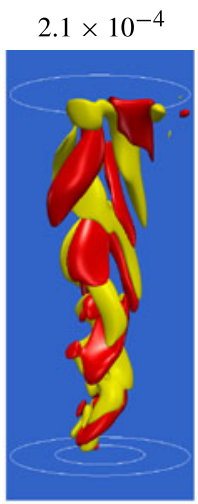

$2.7 \times 10^{-4}$

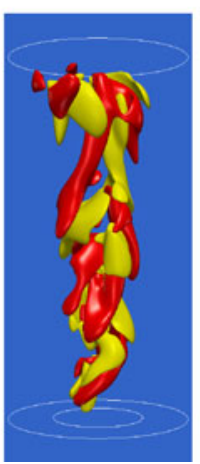

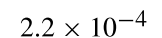
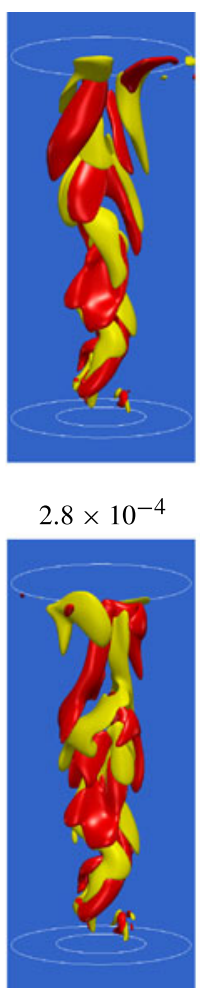

$2.3 \times 10^{-4}$

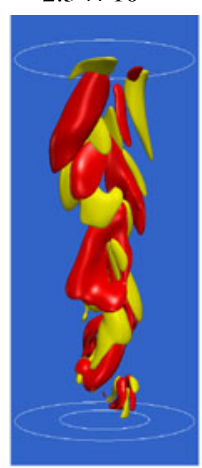

$2.9 \times 10^{-4}$

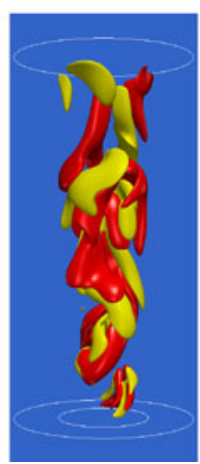

$2.4 \times 10^{-4}$

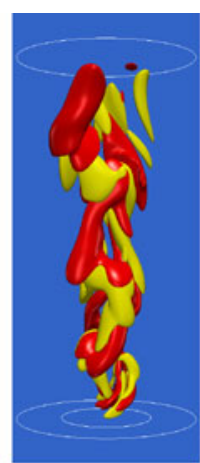

$3.0 \times 10^{-4}$

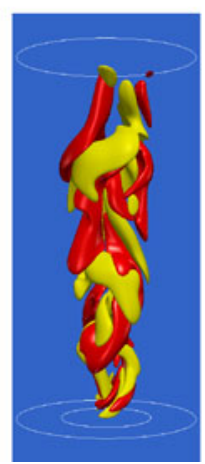

$2.5 \times 10^{-4}$

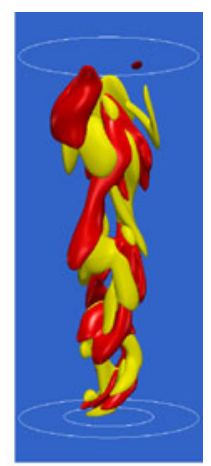

$3.1 \times 10^{-4}$

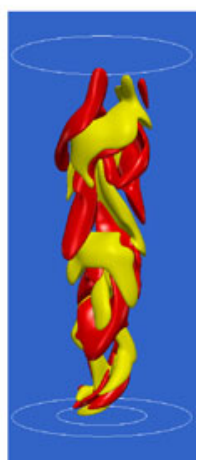

FIGURE 19. (Colour online) Isosurfaces of axial vorticity (levels $\zeta= \pm 5 \times 10^{4}$, red/dark grey is positive and yellow/light grey is negative) of the plume at $R a=3 \times 10^{8}$, at times as indicated, covering two 'puff' periods. The images have been cropped to show only the central plume region.

\section{Discussion and conclusions}

We have presented a detailed study of the transition to turbulence of a thermal plume of Prandtl number $\sigma=7$ driven by a hot spot at the bottom of a cylinder of moderate aspect ratio. The plume becomes turbulent after a succession of symmetrybreaking bifurcations. The first bifurcation is a Hopf bifurcation to an axisymmetric plume periodically releasing puffs of warm fluid that propagate along the plume. The next bifurcation breaks the $O(2)$ axisymmetry. Generally, the breaking of $O(2)$ may result in either standing or rotating waves. For $\sigma=7$ we obtain standing waves meandering in a fixed meridional plane whose azimuthal orientation is arbitrary and selected by initial conditions, in agreement with the experimental observations of Kimura \& Bejan (1983). In the present study, the first bifurcation preserves the axisymmetry, and the second bifurcation preserves the reflection symmetry along a meridional plane, resulting in a continuous family of solutions corresponding to the different azimuthal orientations of this plane.

Dynamical systems theory combined with our numerical simulations suggests that after breaking $O(2)$ symmetry, and in a small range of Rayleigh number, a subsequent bifurcation breaking the reflection symmetry takes place. The bifurcated solution still exhibits a meandering behaviour in a fixed meridional plane, but the reflection 
symmetry with respect to this plane is only approximately fulfilled. However, the temporally invariant torus on which this solution evolves is reflection-invariant as a set. In the present problem, the breaking of the reflection symmetry does not result in a drift of the meridional plane and therefore there is no swirl generation. The breaking of the reflection symmetry seems to be physically related to the collision of the puffs with the top endwall.

On further increasing the Rayleigh number, the invariant torus breaks up resulting in temporally chaotic dynamics and the flow becomes turbulent. Even in this chaotic regime, the meridional plane where the plume undergoes its chaotic wobbling and flickering motion remains temporally invariant for a long time. However, at sufficiently large Rayleigh numbers it is possible that this meridional plane changes orientation from time to time in a random way, much like the so-called spontaneous diffusive meandering of the large-scale circulation in high-Rayleigh-number convection in cylinders (see the review article Ahlers et al. 2009).

The transition process of the thermal plume studied here has much in common with the transition process of the viscous wake of a sphere (Tomboulides \& Orszag 2000), with the main difference being that in the sphere problem (for the parameter regimes studied), the first instability from the steady axisymmetric state is a pitchfork-ofrevolution breaking the $S O(2)$ axisymmetry of the $O(2)$ system via an $m=1$ azimuthal mode, rendering the wake to consist of a pair of counter-rotating streamwise-oriented vortices that are mirror images in a meridional plane (i.e. the $Z_{2}$ symmetry of $O(2)$ is retained), with the orientation of the plane in azimuth being arbitrary. This state becomes unsteady for higher speeds of the flow past the sphere, non-dimensionally measured by the Reynolds number $R e$. The $m=1$ time-periodic state consists of vortex loops periodically shed from the sphere, and their structure is very similar to our flickering plume state (even though our flickering plume is quasi-periodic). At higher $R e$, the periodic wake of the sphere becomes chaotic as two things occur: (i) the orientation of the meridional symmetry plane changes erratically in time; and (ii) the flow loses the $Z_{2}$ reflection symmetry both on average and locally in time (Mittal, Wilson \& Najjar 2001). Similar things may happen with the plume studied here, but to address this issue, additional detailed explorations and very long (and costly) time integrations are needed. Of course, these two flow problems just illustrate two possible transition scenarios for an $O(2)$-symmetric problem, in which the primary instabilities in one case (plume) first break time invariance and then $S O(2)$ while retaining $Z_{2}$, and in the other (sphere) the $S O(2)$ is first broken retaining both time invariance and $Z_{2}$. Another possibility is that the $Z_{2}$ is broken early on leading to rotating waves that precess in either sense depending on initial conditions or modulated rotating waves if the system first breaks time invariance while retaining $O(2)$. These have been observed in the coiling instabilities of liquid threads (Maleki et al. 2004), and of more direct relevance to the present problem, in the instabilities of a thermal plume driven by localized heating in a stably stratified environment (Torrance 1979).

The detailed analysis of a thermal plume presented here illustrates the importance of symmetries in fluid mechanics, and their strong influence on the bifurcations that the flow undergoes. In fact, problems that seems unrelated, such as the wake past a sphere and the thermal plume considered in this study, show a striking similarity. The structure of the plume shown in figure 19 strongly resembles the wake of a sphere because the symmetries in both problems are the same, although the sequence of symmetry-breaking bifurcations is slightly different.

Not only are the symmetries of individual solutions important, but to some extent the symmetries of the invariant sets on which the solutions are located (e.g. tori 
and strange attractors) are more important. These symmetric invariant sets persist even in turbulent flows, and manifest themselves as symmetries verified on average. They organize the flow, producing striking visual signals, such as the meridional plane reflection symmetry in our plumes. Two well-known examples are found in the Taylor-Couette flows. When the outer cylinder is at rest, the axisymmetric Taylor vortices can be observed in fully turbulent flow, and when the cylinders counter-rotate, the turbulent spiral flow shows bands of laminar and turbulent flow that have a beautiful helical symmetry on average (Coles 1965; Andereck, Liu \& Swinney 1986; Dong 2007; Meseguer et al. 2009; Dong \& Zheng 2011). Therefore, the analysis of the symmetries of invariant sets can play an important role in the description of the turbulence.

\section{Acknowledgements}

This work was supported in part by the US National Science Foundation grant DMS-0922864 and the Spanish Ministry of Education and Science grant FIS200908821 .

\section{Supplementary movies}

Supplementary movies are available at http://dx.doi.org/10.1017/jfm.2013.537.

\section{REFERENCES}

Ahlers, G. 2009 Turbulent convection. Physics 2, 74.

Ahlers, G., Grossman, S. \& Lohse, D. 2009 Heat transfer and large-scale dynamics in turbulent Rayleigh-Bénard convection. Rev. Mod. Phys. 81, 503-537.

Altmeyer, S., Do, Y., Marques, F. \& Lopez, J. M. 2012 Symmetry-breaking Hopf bifurcations to 1-, 2-, and 3-tori in small-aspect-ratio counterrotating Taylor-Couette flow. Phys. Rev. E 86, 046316.

Andereck, C. D., LiU, S. S. \& Swinney, H. L. 1986 Flow regimes in a circular Couette system with independently rotating cylinders. J. Fluid Mech. 164, 155-183.

Armbruster, D., Guckenheimer, J. \& Holmes, P. 1988 Heteroclinic cycles and modulated travelling waves in systems with $O(2)$ symmetry. Physica D 29, 257-282.

BAines, W. D. \& TURner, J. S. 1969 Turbulent buoyant convection from a source in a confined region. J. Fluid Mech. 37, 51-80.

Batchelor, G. K. \& Gill, A. E. 1962 Analysis of the stability of axisymmetric jets. J. Fluid Mech. 14, 529-551.

Cetegen, B. M., Dong, Y. \& Soteriou, M. C. 1998 Experiments on stability and oscillatory behaviour of planar buoyant plumes. Phys. Fluids 10, 1658-1665.

Chossat, P. \& LAUterbach, R. 2000 Methods in Equivariant Bifurcations and Dynamical Systems. World Scientific.

Coles, D. 1965 Transition in circular Couette flow. J. Fluid Mech. 21, 385-425.

Colomer, J., Boubnov, B. M. \& Fernando, H. J. S. 1999 Turbulent convection from isolated sources. Dyn. Atmos. Oceans 30, 125-148.

CRAWford, J. D. \& KNobloch, E. 1988 Symmetry-breaking bifurcations in $O(2)$ maps. Phys. Lett. A 128, 327-331.

Crawford, J. D. \& KNObloch, E. 1991 Symmetry and symmetry-breaking bifurcations in fluid dynamics. Annu. Rev. Fluid Mech. 23, 341-387.

Dangelmayr, G. \& Knobloch, E. 1987 The Takens-Bogdanov bifurcation with $O(2)$ symmetry. Phil. Trans. R. Soc. Lond. A 322, 243-279.

DesJardin, P. E., O’Hern, T. J. \& Tieszen, S. R. 2004 Large eddy simulation and experimental measurements of the near-field of a large turbulent helium plume. Phys. Fluids 16, 1866-1883. 
Desrayaud, G. \& Lauriat, G. 1993 Unsteady confined buoyant plumes. J. Fluid Mech. 252, 617-646.

Do, Y., Lopez, J. M. \& Marques, F. 2010 Optimal harmonic response in a confined Bödewadt boundary layer flow. Phys. Rev. E 82, 036301.

Dong, S. 2007 Direct numerical simulation of turbulent Taylor-Couette flow. J. Fluid Mech. 587, 373-393.

Dong, S. \& ZHENG, X. 2011 Direct numerical simulation of spiral turbulence. J. Fluid Mech. 668, $150-173$.

Elicer-Cortés, J. C., Navia, A., Boyer, D., Pavageau, M. \& Hernández, R. H. 2006 Experimental determination of preferred instability modes in a mechanically excited thermal plume by ultrasound scattering. Exp. Therm. Fluid Sci. 30, 355-365.

FAnNelop, T. K. \& WebBeR, D. M. 2003 On buoyant plumes rising from area sources in a calm environment. J. Fluid Mech. 497, 319-334.

FAY, J. A. 1973 Buoyant plumes and wakes. Annu. Rev. Fluid Mech. 5, 151-160.

Fornberg, B. 1998 A Practical Guide to Pseudospectral Methods. Cambridge University Press.

GeBhART, B. 1973 Instability, transition, and turbulence in buoyancy-induced flows. Annu. Rev. Fluid Mech. 5, 213-246.

Golubitsky, M. \& Stewart, I. 2002 The Symmetry Perspective: From Equilbrium to Chaos in Phase Space and Physical Space. Birkhäuser.

Hama, F. R. 1962 Streaklines in a perturbed shear flow. Phys. Fluids 5, 644-650.

Haragus, M. \& Iooss, G. 2011 Local Bifurcations, Center Manifolds, and Normal Forms in Infinite-Dimensional Dynamical Systems. Springer.

Hu, H. H. \& Patankar, N. 1995 Non-axisymmetric instability of core-annular flow. J. Fluid Mech. 290, 213-224.

Hughes, S. \& RANDriamampianina, A. 1998 An improved projection scheme applied to pseudospectral methods for the incompressible Navier-Stokes equations. Intl J. Numer. Meth. Fluids 28, 501-521.

JALURIA, Y. \& GeBhaRT, B. 1975 On the buoyancy-induced flow arising from a heated hemisphere. Intl J. Heat Mass Transfer 18, 415-431.

JIANG, X. \& LUO, K. H. 2000 Direct numerical simulation of the puffing phenomenon of an axisymmetric thermal plume. Theor. Comput. Fluid Dyn. 14, 55-74.

KAYE, N. B. \& Hunt, G. R. 2010 The effect of floor heat source area on the induced airflow in a room. Build. Environ. 45, 839-847.

KimurA, S. \& BeJAN, A. 1983 Mechanism for transition to turbulence in buoyant plume flow. Intl J. Heat Mass Transfer 26, 1515-1532.

KNOBLOCH, E. 1986 On the degenerate Hopf bifurcation with $O(2)$ symmetry. In Multiparameter Bifurcation Theory (ed. M. Golubitsky \& J. Guckenheimer), Contemporary Mathematics, vol. 56, pp. 193-201. American Mathematical Society.

KNOBLOCH, E. 1996 Symmetry and instability in rotating hydrodynamic and magnetohydrodynamic flows. Phys. Fluids 8, 1446-1454.

KrUPA, M. 1990 Bifurcations of relative equilibria. SIAM J. Math. Anal. 21, 1453-1486.

Linden, P. F. 2000 Convection in the environment. In Perspectives in Fluid Dynamics (ed. G. K. Batchelor, H. K. Moffatt \& M. G. Worster), pp. 289-345. Cambridge University Press.

List, E. J. 1982 Turbulent jets and plumes. Annu. Rev. Fluid Mech. 14, 189-212.

Lopez, J. M. \& MARQUeS, F. 2009 Centrifugal effects in rotating convection: nonlinear dynamics. J. Fluid Mech. 628, 269-297.

Lopez, J. M. \& MARQUeS, F. 2010 Sidewall boundary layer instabilities in a rapidly rotating cylinder driven by a differentially co-rotating lid. Phys. Fluids 22, 114109.

Lopez, J. M. \& MARques, F. 2011 Instabilities and inertial waves generated in a librating cylinder. J. Fluid Mech. 687, 171-193.

Maleki, M., Habibi, M., Golestanian, R., Ribe, N. M. \& Bonn, D. 2004 Liquid rope coiling on a solid surface. Phys. Rev. Lett. 93, 214502.

Marques, F., Lopez, J. M. \& Blackburn, H. M. 2004 Bifurcations in systems with $Z_{2}$ spatio-temporal and $O(2)$ spatial symmetry. Physica D 189, 247-276. 
Marques, F., Mercader, I., Batiste, O. \& Lopez, J. M. 2007 Centrifugal effects in rotating convection: axisymmetric states and three-dimensional instabilities. J. Fluid Mech. 580, 303-318.

Massaguer, J. M., Mercader, I. \& Net, M. 1990 Nonlinear dynamics of vertical vorticity in low-Prandtl number thermal convection. J. Fluid Mech. 214, 579-597.

MAXWORThy, T. 1999 The flickering candle: transition to a global oscillation in a thermal plume. J. Fluid Mech. 390, 297-323.

Mercader, I., Batiste, O. \& Alonso, A. 2010 An efficient spectral code for incompressible flows in cylindrical geometries. Comput. Fluids 39, 215-224.

Mercader, I., Net, M. \& Falqués, A. 1991 Spectral methods for high order equations. Comput. Meth. Appl. Mech. Engng 91, 1245-1251.

Meseguer, A., Mellibovsky, F., Avila, M. \& Marques, F. 2009 Instability mechanisms and transition scenarios of spiral turbulence in Taylor-Couette flow. Phys. Rev. E 80, 046315.

MitTAL, R., Wilson, J. J. \& NAJJAR, F. M. 2001 Symmetry properties of the transitional sphere wake. AIAA J. 40, 579-582.

Morton, B. R., TAylor, G. \& Turner, J. S. 1956 Turbulent gravitational convection from maintained and instantaneous sources. Proc. R. Soc. Lond. A 234, 1-23.

Murphy, J. O. \& LOPEZ, J. M. 1984 The influence of vertical vorticity on thermal convection. Austral. J. Phys. 37, 41-62.

NaGAtA, W. 1986 Unfoldings of degenerate Hopf bifurcations with $O(2)$ symmetry. Dyn. Stab. Syst. 1, 125-157.

Navarro, M. C. \& Herrero, H. 2011 Vortex generation by a convective instability in a cylindrical annulus non-homogeneously heated. Physica D 240, 1181-1188.

Orszag, S. A. \& PAtera, A. T. 1983 Secondary instability of wall-bounded shear flows. J. Fluid Mech. 128, 347-385.

Pham, M. V., Plourde, F. \& Doan, S. K. 2007 Direct and large-eddy simulations of a pure thermal plume. Phys. Fluids 19, 125103.

Pham, M. V., Plourde, F. \& Kim, S. D. 2005 Three-dimensional characterization of a pure thermal plume. J. Heat Transfer 127, 624-636.

Plourde, F., Pham, M. V., Kim, S. D. \& Balachandar, S. 2008 Direct numerical simulations of a rapidly expanding thermal plume: structure and entrainment interaction. J. Fluid Mech. 604, 99-123.

Siggia, E. D. 1994 High Rayleigh number convection. Annu. Rev. Fluid Mech. 26, 137-168.

Soteriou, M. C., Dong, Y. \& Cetegen, B. M. 2002 Lagrangian simulation of the unsteady near field dynamics of planar buoyant plumes. Phys. Fluids 14, 3118-3140.

Tomboulides, A. G. \& Orszag, S. A. 2000 Numerical investigation of transitional and weak turbulent flow past a sphere. J. Fluid Mech. 416, 45-73.

TORRANCE, K. E. 1979 Natural convection in thermally stratified enclosures with localized heating from below. J. Fluid Mech. 95, 477-495.

Torrance, K. E., Orloff, L. \& Rockett, J. A. 1969 Experiments on natural convection in enclosures with localized heating from below. J. Fluid Mech. 36, 21-31.

Torrance, K. E. \& RocketT, J. A. 1969 Numerical study of natural convection in an enclosure with localized heating from below - creeping flow to the onset of laminar instability. J. Fluid Mech. 36, 33-54.

Turner, J. S. 1969 Buoyant plumes and thermals. Annu. Rev. Fluid Mech. 1, 29-44.

UlUCAKI, M. E. 1996 Turbulent natural convection in an enclosure with localized heating from below. Exp. Heat Transfer 9, 305-321.

Williams, T. C., Shaddix, C. R., Schefer, R. W. \& Desgroux, P. 2007 The response of buoyant laminar diffusion flames to low-frequency forcing. Combust. Flame 151, 676-684.

Woods, A. W. 2010 Turbulent plumes in nature. Annu. Rev. Fluid Mech. 42, 391-412.

YANG, H. Q. 1992 Buckling of a thermal plume. Intl J. Heat Mass Transfer 35, 1527-1532. 